\title{
HIDE AND SEEK BETWEEN ANDROMEDA'S HALO, DISK, AND GIANT STREAM*
}

\author{
Gisella Clementini $^{1}$, Rodrigo Contreras Ramos ${ }^{1,2}$, Luciana Federici $^{1}$, Giulia Macario ${ }^{1,2}$, Giacomo Beccari $^{3}$, \\ Vincenzo Testa $^{4}$, Michele Cignoni ${ }^{2}$, Marcella Marconi ${ }^{5}$, Vincenzo Ripepi ${ }^{5}$, Monica Tosi ${ }^{1}$, Michele Bellazzini $^{1}$, \\ Flavio Fusi Pecci ${ }^{1}$, Emiliano Diolaiti ${ }^{1}$, Carla Cacciari $^{1}$, Bruno Marano ${ }^{2}$, Emanuele Giallongo ${ }^{4}$, \\ Roberto Ragazzoni ${ }^{6}$, Andrea Di Paola ${ }^{4}$, Stefano Gallozzi ${ }^{4}$, and Riccardo Smareglia ${ }^{7}$ \\ ${ }^{1}$ INAF, Osservatorio Astronomico di Bologna, Bologna, Italy; gisella.clementini@oabo.inaf.it \\ ${ }^{2}$ Dipartimento di Astronomia, Università di Bologna, Bologna, Italy \\ ${ }^{3}$ European Southern Observatory, 85748 Garching bei Munchen, Germany \\ ${ }_{5}^{4}$ INAF, Osservatorio Astronomico di Roma, Monteporzio, Italy \\ ${ }^{5}$ INAF, Osservatorio Astronomico di Capodimonte, Napoli, Italy \\ ${ }^{6}$ INAF, Osservatorio Astronomico di Padova, Padova, Italy \\ ${ }^{7}$ INAF, Osservatorio Astronomico di Trieste, Trieste, Italy \\ Received 2011 February 14; accepted 2011 September 6; published 2011 November 18
}

\begin{abstract}
Photometry in $B, V$ (down to $V \sim 26$ mag) is presented for two $23^{\prime} \times 23^{\prime}$ fields of the Andromeda galaxy (M31) that were observed with the blue channel camera of the Large Binocular Telescope during the Science Demonstration Time. Each field covers an area of about $5.1 \times 5.1 \mathrm{kpc}^{2}$ at the distance of M31 ( $\left.\mu_{\mathrm{M} 31} \sim 24.4 \mathrm{mag}\right)$, sampling, respectively, a northeast region close to the M31 giant stream (field S2) and an eastern portion of the halo in the direction of the galaxy minor axis (field H1). The stream field spans a region that includes Andromeda's disk and giant stream, and this is reflected in the complexity of the color-magnitude diagram of the field. One corner of the halo field also includes a portion of the giant stream. Even though these demonstration time data were obtained under non-optimal observing conditions, the $B$ photometry, which was acquired in time-series mode, allowed us to identify 274 variable stars (among which 96 are bona fide and 31 are candidate RR Lyrae stars, 71 are Cepheids, and 16 are binary systems) by applying the image subtraction technique to the selected portions of the observed fields. Differential flux light curves were obtained for the vast majority of these variables. Our sample mainly includes pulsating stars that populate the instability strip from the Classical Cepheids down to the RR Lyrae stars, thus tracing the different stellar generations in these regions of M31 down to the horizontal branch of the oldest $(t \sim 10 \mathrm{Gyr})$ component.
\end{abstract}

Key words: galaxies: individual (M31) - galaxies: stellar content - stars: variables: Cepheids - stars: variables: RR Lyrae

Online-only material: color figures, extended figures, machine-readable table

\section{INTRODUCTION}

The Andromeda galaxy, which is our nearest giant neighbor, is the best place to study the structure, formation, and evolution of a massive spiral and to get hints on whether the merger/ accretion or the cloud collapse is the dominant mechanism in the formation of a giant spiral. Our external view of the system is less affected by selection and line-of-sight effects that make it difficult to perform a homogeneous global study of the Milky Way (MW). As a result, the structures observed in M31 are easier to study, thus making Andromeda the best current laboratory for investigating faint stellar structures and merging events occurring around large galaxies.

Van den Bergh $(2000,2006)$ suggested that M31 originated as an early merger of two or more relatively massive metalrich progenitors. This would account for the wide range in metallicity (Durrell et al. 2001; Stephens et al. 2001; Bellazzini et al. 2003) and age (Brown et al. 2003) observed in the M31 halo compared with the MW. M31 hosts spectacular signatures of present and past merging events, such as the giant tidal stream (Ibata et al. 2001) that extends several degrees from the center of the galaxy (McConnachie et al. 2003) and the arc-like overdensity connecting the galaxy to its dwarf elliptical

\footnotetext{
* Based on data acquired using the blue channel camera of the Large Binocular Telescope (LBT/LBC-blue).
}

companion NGC 205 (McConnachie et al. 2004). Metallicity, luminosity, and alignment with M32 are consistent with the M31 stream being tidally extracted from M32. A number of earlier papers following the stream discovery did indeed speculate that M32 might be the source of the stream (Ibata e al. 2001; Choi et al. 2002; Ferguson et al. 2002). The low-velocity dispersion $\left(11 \mathrm{~km} \mathrm{~s}^{-1}\right)$ supports the notion that the giant stream is a coherent interaction remnant. However, the Keck/DEep Imaging MultiObject Spectrograph spectroscopic studies (Font et al. 2006), the models for the progenitor, and the $\mathrm{N}$-body studies of its tidal disruption tend to argue against M32 as a stream progenitor (Fardal et al. 2008). The infrared photometry of the M31 disk obtained by the Infrared Astronomical Satellite (Habing et al. 1984) and by the Spitzer Space Telescope (Barmby et al. 2006) revealed two rings of star formation off-centered from the M31 nucleus (Block et al. 2006 and references therein). The two rings seem to be density waves propagating in the disk. Beccari et al. (2007) unveiled the near-ultraviolet view of the M31 inner ring using the blue channel camera of the Large Binocular Telescope (LBT/LBC-blue). Numerical simulations show that both rings may result from a companion galaxy plunging head-on through the center of the M31 disk about $210 \mathrm{Myr}$ ago (Block et al. 2006).

Ferguson et al. (2002) and Ibata et al. (2007) presented the first panoramic views of the Andromeda galaxy based on the deep Isaac Newton Telescope (INT) and the Canada-France-Hawaii 
Telescope (CFHT) photometric observations that cover, respectively, the galaxy's inner $55 \mathrm{kpc}$ and the southern quadrant out to about $150 \mathrm{kpc}$, with an extension that reaches M33 at a distance of about $200 \mathrm{kpc}$. Their data show the whole giant stream, including its extensions and also reveal a multitude of streams, arcs, and many other large-scale structures of low surface brightness, as well as two new M31 dwarf companions (And XV and And XVI). Twelve dwarf galaxies were known to be M31 companions until 2004, among which only six dwarf spheroid galaxies (dSphs) and an additional seventeen new M31 satellites were discovered in the last five to six years, mainly based on the INT and the CFHT data. The most recent census is reported by Richardson et al. (2011) along with the discovery of five new M31 dSph satellites, And XXIII-XXVII, as a result from the second year of data from the "PanAndromeda Archaeological Survey" (PAndAS; McConnachie et al. 2009). Started in 2008 August, the PAndAS survey is extending the global view of M31 and that of its companion M33 by collecting data with MegaPrime/MegaCam on the $3.6 \mathrm{~m} \mathrm{CFHT}$, for a total area of $300 \mathrm{deg}^{2}$ and out to a maximum projected radius from M31's center of about $150 \mathrm{kpc}$. This survey will likely detect several other M31 faint satellites.

The primary tool for understanding the formation history of a galaxy over the whole Hubble time is the analysis of color-magnitude diagrams (CMDs) deep enough to reach the main-sequence turnoff (TO) of the oldest populations. The TO of the oldest stars in M31 ( $V \sim 28.5 \mathrm{mag}$ ) is still unreachable by the largest ground-based telescopes, and it requires tens of orbits of the Hubble Space Telescope/Advanced Camera for Surveys $(H S T / A C S)$ time devoted to "tiny" $(3.5 \times 3.7)$ portions of the galaxy (Brown et al. 2003, 2006, 2007, 2008, 2009) to be reached and measured. The shallower survey by Richardson et al. (2008), who obtained CMDs to $V \sim 27.5$ mag for 14 HST/ ACS fields and sampled different substructures around M31, covers a total area that is roughly one-third of that allowed in a single shot by a ground-based telescope like LBT.

Pulsating variable stars offer a powerful alternative tool to trace stars of different ages in a galaxy because variables of different types arise from parent populations of different ages. Specifically, the RR Lyrae stars and the Population II Cepheids (often referred to as Type II Cepheids [T2Cs]), which belong to the oldest stellar population $(t>10 \mathrm{Gyr})$, allow us to trace the star formation history (SFH) back to the first epochs of galaxy formation and, with their mere presence, can provide crucial insight into the timescale and merging episodes that may have led to the assembly of a galaxy. These low-mass variables $\left(M \leqslant \sim 1 M_{\odot}\right.$ ) burn helium in the stellar core and hydrogen in the shell surrounding the core during the horizontal branch (HB, the RR Lyrae) and the post-HB (the T2Cs) evolutionary phases. They are, respectively, about 3 and 4 magnitude brighter and hence are much easier to observe than old $(t>10 \mathrm{Gyr}) \mathrm{TO}$ stars. The typical shape of their light variation, which occurs with periodicities in the range of 0.2 to less than 1 day for the RR Lyrae stars and from 1 to 25 days for T2Cs, makes them much easier to recognize, even when the upper Hertzsprung-Russel (H-R) diagram has overlapping contributions from a complex mix of age and metallicity. The Anomalous Cepheids (ACs) are intermediate-age ( $t \sim 1-2 \mathrm{Gyr})$ variables with periods in the range of $\sim 0.3$ to $\sim 2.0$ days and with mean magnitudes spanning a 1.5-mag range. These variables are intrinsically brighter than the RR Lyrae stars and, when observed in an external stellar system where distance and projection effects can be neglected, they locate themselves along a strip, with the least luminous ones (at periods $\sim 0.30$ days) being about 0.5 mag brighter than the RR Lyrae stars, and the most luminous ones (at periods $\sim 2.0$ days) being about 2.0 mag brighter than the RR Lyrae stars. They appear significantly brighter than the T2Cs at the fixed period. From an evolutionary point of view they are metalpoor $([\mathrm{Fe} / \mathrm{H}] \leqslant-1.7)$ He-burning stars in the post-turnover portion of the zero-age horizontal branch (ZAHB; see Marconi et al. 2004 and references therein) that cross the instability strip (IS) at a higher luminosity than the RR Lyrae variables. Their mass is around $1.5 M_{\odot}$. With an age of a few Gyr, they indeed represent the natural extension of Classical Cepheids (CCs) to lower metallicities and masses (see, e.g., Caputo et al. 2004; Fiorentino et al. 2006 and references therein). Finally, CCs are intermediate-mass (typically from 3 to $12 M_{\odot}$ ) stars that cross the IS during the blueward excursion in the central He-burning phase, also referred to as a blue loop. They have pulsation periods in the range of $\sim 1$ to $\sim 100$ days and an absolute visual magnitude of -2 to -7 mag. With a typical age ranging from a few to a few hundred Myr, they represent excellent tracers of the properties of young Population I stars.

Short- and intermediate-period variables (i.e., periods shorter than a few days) of M31 have never been adequately studied despite their great potential to trace the stellar populations and the star formation histories (SFHs) of nearby galaxies (Mateo 1998, 2000; Saha 1999; Catelan 2004; Clementini et al. 2004). So far, the main limitations have been the size of the telescopes employed in the ground-based surveys (Pritchet \& van den Bergh 1987; Dolphin et al. 2004; Vilardell et al. 2007; Joshi et al. 2010), the limited number of available $H S T$ archive data (Clementini et al. 2001), and the rather small areas of M31 covered by the space observations (Brown et al. 2004; Sarajedini et al. 2009; Jeffery et al. 2011). In their deep HST / ACS survey of the M31 halo, Brown et al. (2004) identified 55 RR Lyrae stars (29 fundamental-mode -RRab-, 25 first overtone - RRc, and one double-mode $-\mathrm{RRd}-$ pulsators) in a 3.5 × 3.7 field along the southeast minor axis of the galaxy. Based on their pulsation properties, Brown et al. concluded that the old population in the Andromeda halo has Oosterhoff-intermediate properties and does not conform to the subdivision in Oosterhoff I (Oo I) and Oosterhoff II (Oo II) types (Oosterhoff 1939) followed by the RR Lyrae stars in the MW field and globular clusters (GCs; see Clementini 2010 and references therein). In this respect, the M31 halo field would thus be different from the MW halo field, and this would point to a different formation/evolution history. However, a different conclusion was reached by Sarajedini et al. (2009) who identified 681 RR Lyrae variables (555 RRab's, and 126 RRc's) based on the HST/ACS observations of two fields near M32, at a projected distance between 4 and $6 \mathrm{kpc}$ from the center of M31, and concluded that these M31 fields have Oo I properties. More recently, Jeffery et al. (2011) have extended Brown et al.'s study of the M31 halo RR Lyrae stars to five additional HST/ACS fields that include: one pointing in the stream, one pointing in the disk, and three pointings in the M31 halo, at distances of $21 \mathrm{kpc}$ (hereafter referred to as halo21) and $35 \mathrm{kpc}$ from the galaxy center (hereafter halo35a and halo35b, respectively; see Figure 1 in the Jeffery et al. paper). They detected 21 RR Lyrae stars in the "disk" field, 24 in the "stream" field, 3 in the "halo21" field, 5 in the "halo35b" field, and none in the "halo35a" field. Field "halo21" is of particular interest to us since it overlaps with our field H1. Jeffery et al. found average periods for the fundamental mode pulsators of 0.583 , 0.560 , and 0.495 days in the disk, stream, halo21, and halo35b fields, respectively, and conclude that the RR Lyrae populations 


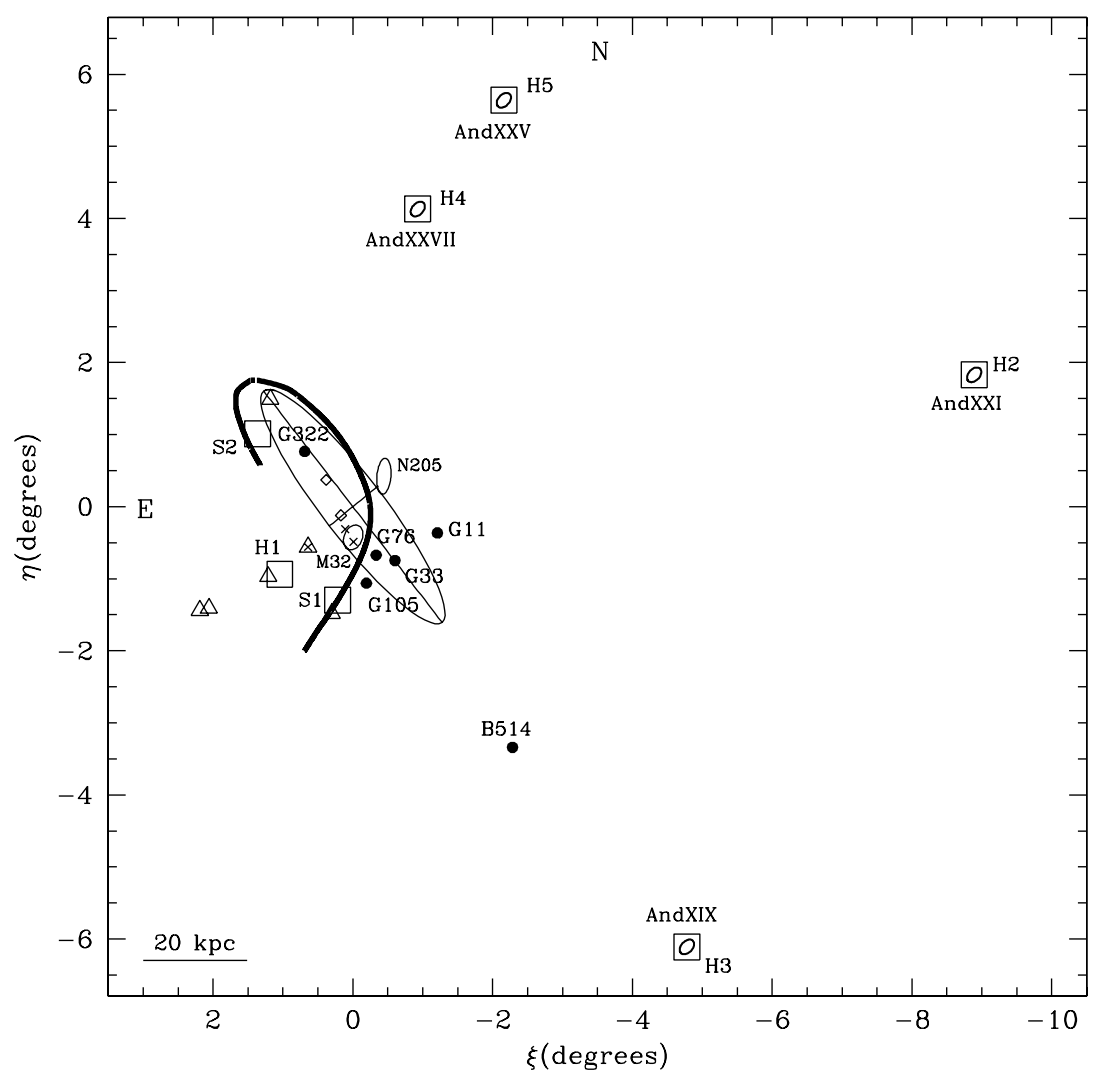

Figure 1. Schematic map of the Andromeda galaxy showing the location of the fields targeted with the LBT/LBC-blue (boxes) and the GCs observed with the HST (filled circles). The heavy solid line shows the approximate location of the M31 giant stream according to Ferguson et al. (2002). Crosses show the centers of the M31 fields studied by Brown et al. (2004) and Sarajedini et al. (2009) using the HST/ACS time-series data. Open triangles show the M31 fields studied with HST/ACS by Jeffery et al. (2011). Diamonds mark the center positions of the M31 fields studied with the ground-based time series observations by Vilardell et al. (2006, 2007) and Joshi et al. (2010).

in these M31 fields appear to be mostly of Oo I. But, how general are all of these results, given the small areas covered by the Brown et al. (2004), Sarajedini et al. (2009), and Jeffery et al. (2011) studies? Another open question is whether there are any RR Lyrae stars in the M31 giant stream and, if any, whether their properties differ from the properties of the variables in the surrounding M31 fields. Jeffery et al. (2011) detected 24 RR Lyrae stars in their "stream" field. These RR Lyrae stars could either belong to the merged satellite, to M32 where RR Lyrae stars are claimed to exist (Alonso-Garcia et al. 2004; Fiorentino et al. 2010), or have formed during the merger, in which case the merging event would have occurred at least $10 \mathrm{Gyr}$ ago. In any case, their pulsation properties could provide hints to identify the progenitor stream stars. According to the average periods in Jeffery et al.'s Table 2, the "stream" RR Lyrae stars seem to be more OoI-like than the variables in the other fields they observed. However, given the small number statistics and the rather limited field of view (FOV) of HST/ACS, this could simply be a statistical artifact. Clearly, sampling of much larger areas is needed to draw any general conclusions on the properties of the M31 RR Lyrae population.

To address the above questions, we are carrying out a longterm project to study the stellar populations of both the constant and variable stars in properly selected fields and GCs of the Andromeda galaxy, as well as in recently discovered M31 dSph satellites. We have used the Wide Field Planetary Camera 2 on board the HST (Cycle 15 HST program GO 11081; PI: G. Clementini) to resolve the cluster stars (see Clementini et al. 2009; Contreras Ramos 2010) and the wide field and lightcollecting capabilities of the LBT to monitor portions of the M31 giant stream and halo, and four of the most extended M31 dSphs: And XIX, And XXI, And XXV, and And XXVII. A European Southern Observatory Large Program (ID: 186.D-2013; PI: G. Clementini) is also in progress at the Gran Telescopio Canarias to study the variable stars and stellar populations of five further M31 dSphs. Figure 1 shows the location of the target fields and GCs (squares and filled circles, respectively) of our HST and LBT observations on a schematic map of the Andromeda galaxy. Also shown in the figure are the fields studied for variability by Brown et al. (2004), Sarajedini et al. (2009), and Jeffery et al. (2011) using the HST/ACS time-series data, and the fields studied for variability by Vilardell et al. $(2006,2007)$ and Joshi et al. (2010) using ground-based facilities.

The study of the cluster variables shows that the RR Lyrae stars in the M31 GCs may have different properties than their MW counterparts (Clementini et al. 2009; Contreras Ramos 2010; R. Contreras Ramos et al. 2011, in preparation). The LBT is an ideal tool for studying the pulsating variable stars in the M31 field and in its dSphs because it reaches the same level of accuracy as the HST studies, but on a much larger area, thus allowing us to attain a statistical significance never reached before for an external galaxy because each LBT field covers an area about 37 times larger than an HST/ACS-WFC field.

The identification and center coordinates of the M31 regions that we are observing with the LBT are provided in Table 1. The stream fields were chosen to monitor both the stream portion that enters into the M31 disk (field S1 in Figure 1) and a region toward the northeast portion of the stream that exits from the disk (field S2 in Figure 1). Fields H1, H2, H3, H4, and $\mathrm{H} 5$ were instead chosen to provide a representation of the 
Table 1

Identification and Coordinates of Our LBT Fields in M31

\begin{tabular}{|c|c|c|c|c|c|c|c|c|}
\hline Name & Object Type & $\begin{array}{c}\alpha \\
(2000)\end{array}$ & $\begin{array}{c}\delta \\
(2000)\end{array}$ & $\begin{array}{c}\xi \\
\text { (deg) }\end{array}$ & $\begin{array}{c}\eta \\
\text { (deg) }\end{array}$ & $\begin{array}{c}D_{\mathrm{M} 31 \mathrm{center}} \\
(\mathrm{kpc})\end{array}$ & $N_{B}{ }^{\mathrm{a}}$ & $N_{V}^{\mathrm{a}}$ \\
\hline $\mathrm{H} 1$ & Halo field & 004813.11 & +401909.4 & 1.04 & -0.94 & 18.9 & 48 & 3 \\
\hline $\mathrm{H} 3$ & And XIX & 001932.1 & +350237.1 & -4.78 & -6.11 & 104.2 & 48 & 46 \\
\hline $\mathrm{H} 4$ & And XXVII & 003727.1 & +452313 & -0.93 & 4.13 & 56.9 & $\mathrm{~b}$ & $\mathrm{~b}$ \\
\hline $\mathrm{S} 2$ & Stream field & 004908.31 & +421609.4 & 1.18 & 1.01 & 20.9 & 59 & 8 \\
\hline
\end{tabular}

Notes.

a Each image corresponds to a 300 s exposure for fields S2, H1, and S1, and to a 420 s exposure for fields H2, H3, H4, and H5.

b Observations scheduled for Fall 2011.

different portions of Andromeda's halo on the opposite sides of the galaxy. In particular, fields $\mathrm{H} 2$ and $\mathrm{H} 3$ are located at about $122 \mathrm{kpc}$ northwest and $104 \mathrm{kpc}$ southwest of the M31 center, respectively, and contain two new M31 dSphs, And XXI (Martin et al. 2009) in field H2 and And XIX (McConnachie et al. 2008) in field H3. Fields $\mathrm{H} 4$ and H5 are located along a filamental structure at about $57 \mathrm{kpc}$ and $81 \mathrm{kpc}$ northwest of the M31 center, respectively, and contain two of the most recently discovered M31 satellites, And XXVII and And XXV (Richardson et al. 2011). Finally, field H1 is at about $19 \mathrm{kpc}$ from the center of M31 in the southeast direction.

In this paper, which is part of our series on the study of variable stars in M31, we present results from pilot observations of fields S2 and H1 obtained during the Science Demonstration Time (SDT) of the LBC-blue mounted at the prime focus of the first unit of LBT (Giallongo et al. 2008). Each of these fields covers a $23^{\prime} \times 23^{\prime}$ area. We have obtained $B, V$ CMDs down to $V \sim 26$ mag for both fields. The large FOV along with the high sensitivity of LBT/LBC-blue allowed us to bridge portions of the M31 disk to traces of the galaxy giant stream in a single shot of field S2. Similarly, the southwest corner of the halo field H1 probably includes the southeast portion of the giant stream. We present results of a search for variable stars in these regions of the Andromeda galaxy. A number of technical problems and rather unfavorable weather/seeing conditions hampered our observing campaign. Nevertheless, using the image subtraction technique we were able to identify and obtain differential flux light curves for a number of CCs with periods in the range of 3 to 10 days, a few candidate ACs and/or, more likely, short-period CCs (spCCs) with periods around 1-2 days, more than $100 \mathrm{RR}$ Lyrae stars, and a number of binary systems in the portions of fields $\mathrm{S} 2$ and $\mathrm{H} 1$ where the image subtraction technique worked out properly.

Observations, data reduction, and the calibration of the photometry are discussed in Section 2. The CMDs of fields $\mathrm{S} 2$ and $\mathrm{H} 1$ are presented in Section 3. Results on the variable stars and the catalog of light curves are presented in Section 4. Finally, a summary and discussion of the results are presented in Section 5.

\section{OBSERVATIONS AND DATA REDUCTION}

$B, V$ photometry of the M31 fields $\mathrm{S} 2$ and $\mathrm{H} 1$ (see Table 1) was obtained with LBT/LBC-blue during $10 \mathrm{hr}$ of SDT of the Blue Channel in 2007 October 11-18. Given the LBT/LBC-blue scale $\left(0.225\right.$ pixel $\left.^{-1}\right)$ and the total FOV $\left(23^{\prime} \times 23^{\prime}\right)$, each of these fields covers an area roughly corresponding to $5.1 \times 5.1 \mathrm{kpc}^{2}$ at the distance of $\mathrm{M} 31\left(\mu_{\mathrm{M} 31} \sim\right.$ $24.4 \mathrm{mag})$. Figure 2 shows the location of fields S2 and $\mathrm{H} 1$ over a $3.5 \times 3.5 \mathrm{deg}^{2}$ image of the Andromeda galaxy obtained from the combination of the 3.4- $\mu, 4.6-\mu, 12-\mu$, and $22-\mu$ fluxes measured by NASA's Wide-field Infrared Survey Explorer (WISE), along with a schematic view of the M31 giant tidal stream.

Both of our fields are contained in the area surveyed by Ferguson et al. (2002) with INT, reaching a limiting magnitude $V \sim 24.5 \mathrm{mag}$, i.e., $1.5 \mathrm{mag}$ shallower than our photometry. These areas are also planned to be observed by PAndAS with limiting magnitude $g_{0} \sim 25.5 \mathrm{mag}$; however, no CMDs of the regions sampled by fields $\mathrm{S} 2$ and $\mathrm{H} 1$ have been published yet. Field H1 contains the two HST/ACS fields of the M31 "Minor axis" observed by Richardson et al. (2008; see their Table 1) and the field "halo21" (see Figure 1) observed by Jeffery et al. (2011).

The RR Lyrae stars in M31 are expected to have average magnitudes around $V \sim 25.3-25.5 \mathrm{mag}$. Taking into account their typical intrinsic colors, amplitudes, and periods $(B-V \sim$ $0.2-0.4, A_{V} \sim 0.3-0.5$ and $0.6-1.2 \mathrm{mag}, P \sim 0.2-1$ day, for first overtone and fundamental mode pulsators, respectively), we aimed at reaching a limiting magnitude of $B \sim 26 \mathrm{mag}$ (corresponding to the minimum light of these variables in M31) in no longer than 15-20 minutes to avoid smearing the light curve and to have an acceptable signal-to-noise ratio $(\mathrm{S} / \mathrm{N})$, even at the light curve minimum. Based on the LBT exposure time calculator, we had estimated that in dark time with a 15 minute exposure and seeing conditions $=1^{\prime \prime}$, we would obtain an $\mathrm{S} / \mathrm{N} \sim 6$ for $B=26 \mathrm{mag}$ and an $\mathrm{S} / \mathrm{N} \sim 9$ for $V=25.5 \mathrm{mag}$.

This would have been perfectly adequate for our purposes. Unfortunately, seeing conditions varied significantly during our observing run, ranging from 0.8 to 2 ".7. We also experienced problems with the focus and tracking of the telescope during these early phases of LBT operation, which did not allow us to make individual exposures longer than $300 \mathrm{~s}$. Our observations, which were acquired in time-series mode, consist of $59 \mathrm{~B}$ and $8 V$ frames of field $\mathrm{S} 2$, and $48 B$ and $3 V$ frames of field $\mathrm{H} 1$, each frame corresponding to a $300 \mathrm{~s}$ exposure, and we obtained an $\mathrm{S} / \mathrm{N} \sim 2$ for $B \sim 26 \mathrm{mag}$ in our best image at FWHM $\sim 0$ '! 8 . Notwithstanding the unfavorable weather and technical conditions, we obtained $30 \mathrm{~B}$ and $6 \mathrm{~V}$ images of field $\mathrm{S} 2$, and $33 B$ and $1 V$ images of field $\mathrm{H} 1$ with FWHM $<1^{\prime \prime} .3$, which, after using the image subtraction technique (ISIS; Alard 2000), allowed us to identify candidate variable stars as faint as $V \sim 25.5$ mag in the portions of fields $\mathrm{S} 2$ and $\mathrm{H} 1$ less affected 


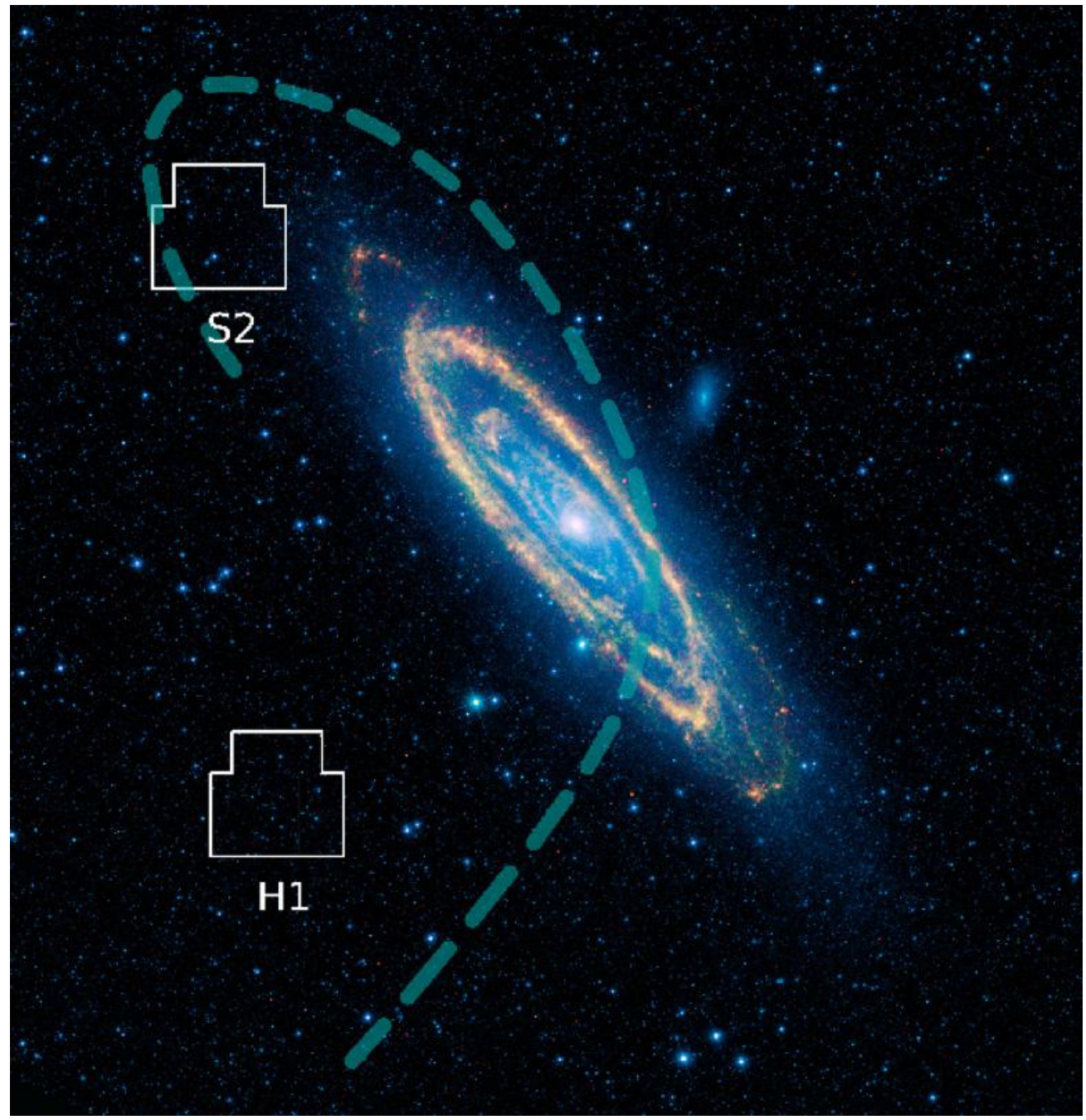

Figure 2. $3.5 \times 3.5 \mathrm{deg}^{2}$ image of the Andromeda galaxy obtained from the combination of the 3.4- $\mu, 4.6-\mu, 12-\mu$, and $22-\mu$ fluxes, measured by the NASA's Wide-field Infrared Survey Explorer (WISE; Image Credit: NASA/JPL-Caltech/UCLA). It shows the location of fields S2 and H1 and a schematic view of the M31 giant tidal stream (heavy dashed line).

(A color version of this figure is available in the online journal.)

by optical distortions, where we succeeded in running ISIS. It should also be noted that the $V$ images of both fields $\mathrm{S} 2$ and $\mathrm{H} 1$ were accidentally trimmed during the readout of the CCDs; as a consequence, the upper 500 pixels of each CCD in the $V$ images were lost.

Pre-reduction of the entire data set (bias-subtraction and flat-fielding) through the LBC-dedicated pipeline was provided by the LBC team. ${ }^{8}$ Point-spread function (PSF) photometry of the pre-reduced images of each chip of the LBC mosaic was then performed with DoPHOT (Schechter et al. 1993) on the two images obtained in the best observing conditions $\left(1 B\right.$ and $1 V$ with the FWHM $\sim 00^{\prime \prime} 8-1^{\prime \prime}$ for each of the two fields) to produce the CMDs. This package allowed us to model the stellar PSF, which varies significantly along each CCD of our LBC frames, much more efficiently than DAOPHOT. On the other hand, our attempt to use DAOPHOTII/ALLSTAR/ ALLFRAME (Stetson 1987, 1994) to process the individual time-series data and produce light curves on a magnitude scale for the variable stars often failed due to both the geometric distortions and the poor FWHM of the vast majority of our frames. For this reason we obtained light curves on a magnitude scale only for a very limited number of variable stars located in small portions of the frames where DAOPHOTII/ALLSTAR/ ALLFRAME ran successfully. A Two Micron All Sky Survey catalog ${ }^{9}$ was used to identify astrometric standards in the LBC FOV. More than a 1000 stars were used to find an astrometric solution for each of the LBC CCDs. Accuracy of the derived coordinates is on the order of $\sim 0^{\prime \prime} 3-0^{\prime \prime} 4$ (rms) in both the right ascension and the declination. The absolute photometric calibration of the $\mathrm{S} 2$ and $\mathrm{H} 1$ photometry was obtained using a set of 192 local secondary standard stars with $B, V$ photometry in the Johnson-Cousins system, which was extracted from the Massey et al. (2006) catalog, and falls in the region of field $\mathrm{S} 2$ covered by CCD 1. Aperture corrections were separately calculated for each of the four CCD mosaics of fields S2 and H1 by performing aperture photometry in each photometric band with the SExtractor package (Bertin \& Arnouts 1996). They are provided in Table 2 . The derived calibration equations are

$$
B=b-0.0635(b-v)+27.78-K_{b} X_{b}
$$


Table 2

Aperture Corrections for the Four CCD Mosaic Image of Field S2 (Upper Part) and H1 (Lower Part)

\begin{tabular}{lcc}
\hline \hline Chip & Field S2 & \\
\hline & $B$ & $V$ \\
CCD 1 & -0.236 & -0.301 \\
CCD 2 & -0.251 & -0.304 \\
CCD 3 & -0.244 & -0.254 \\
CCD 4 & -0.229 & -0.216 \\
\hline & Field H1 & \\
\hline & $B$ & \\
CCD 1 & -0.216 & -0.134 \\
CCD 2 & -0.211 & -0.168 \\
CCD 3 & -0.222 & -0.127 \\
CCD 4 & -0.232 & -0.109 \\
\hline
\end{tabular}

Note. Corrections correspond to aperture minus PSF magnitudes.

and

$$
V=v+0.0107(b-v)+28.12-K_{v} X_{v},
$$

where $B$ and $V$ are the standard magnitudes and $b, v$ are the instrumental magnitudes normalized to $1 \mathrm{~s}$ and corrected for aperture corrections using the values given in Table $2 . K_{b}$ and $K_{v}$ are the extinction coefficients in $B$ and $V$ for which we adopted values of 0.22 and $0.15 \mathrm{mag}$, respectively, as provided on the LBC commissioning Web page (available at http://lbc.oa-roma.inaf.it/commissioning/standards.html). Typical internal errors of our photometry for non-variable stars at the level of the M31 HB ( $V \sim 25.5 \mathrm{mag}$ ) are $\sigma_{V}=0.17 \mathrm{mag}$ and $\sigma_{B}=0.26 \mathrm{mag}$, respectively, as provided by the DoPHOT reduction of individual $B, V$ images corresponding to $300-\mathrm{s}$ exposures.

\section{COLOR-MAGNITUDE DIAGRAMS}

Figures 3 and 4 show the $V, B-V$ CMDs of the four CCD mosaics of fields $\mathrm{S} 2$ and $\mathrm{H} 1$, respectively, obtained at the end of the reduction and calibration processes from the DoPHOT photometry of pairs of $B, V$ images of each field, each corresponding to $300 \mathrm{~s}$ exposures obtained with FWHM of about $0.8-1{ }^{\prime \prime} .0$. The photometric catalogs producing these CMDs were cleaned from stars with photometric errors larger than twice the mean error at each magnitude and by manually removing "spurious stars" produced by ghosts and spikes of saturated sources and background galaxies. In each figure, the CMDs are arranged according to the geometry of the four CCDs composing the LBC-blue mosaic, and each CCD was divided into two equal parts: north and south parts for CCDs 1,2 , and
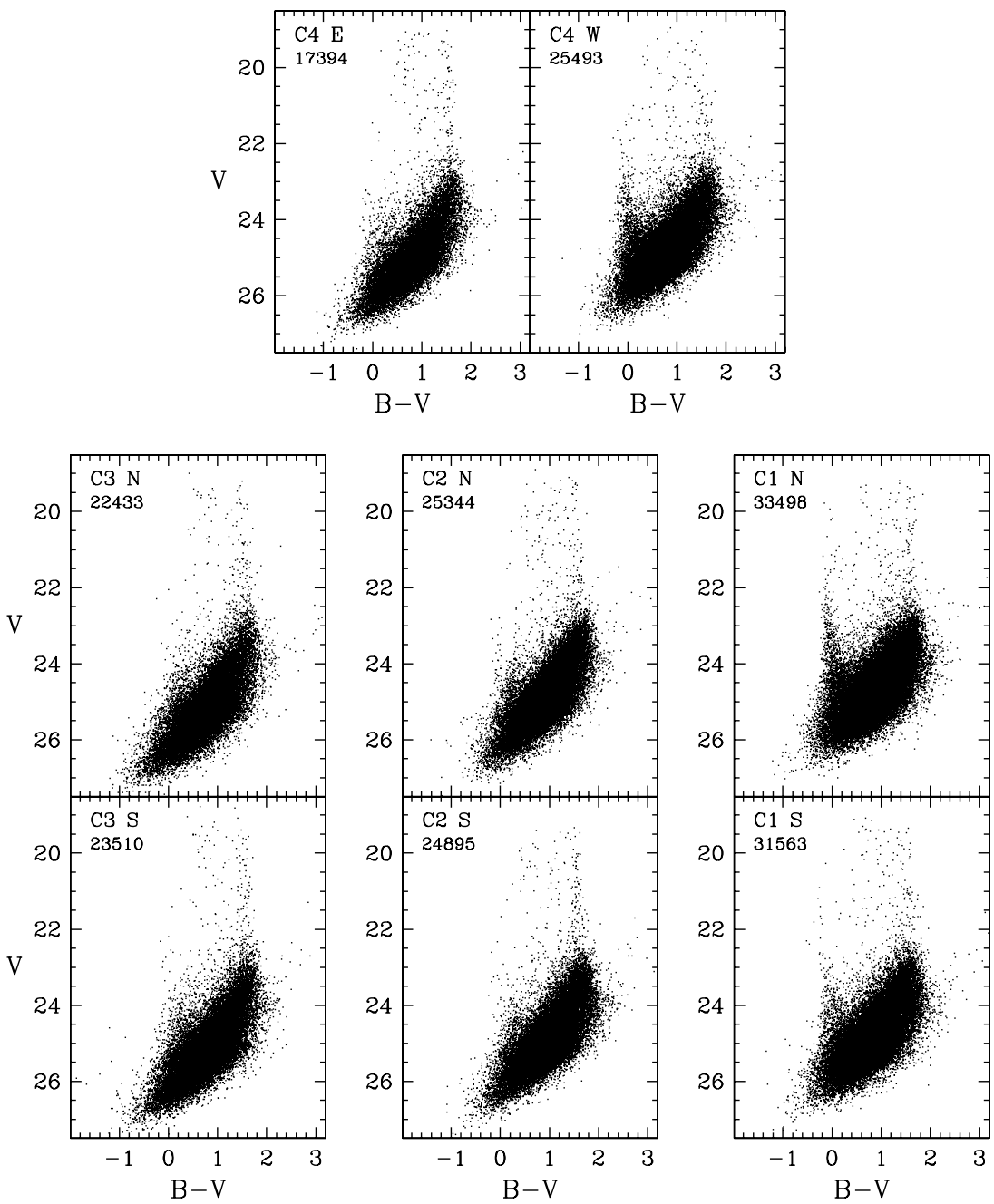

Figure 3. $V, B-V$ CMDs of field $\mathrm{S} 2$, from a pair of $B, V$ images with a $300 \mathrm{~s}$ exposure time; obtained in optimal observing conditions (FWHM $\sim 0$ '. $8-1^{\prime \prime}$. 0 ). Each panel shows the number of stars displayed. 

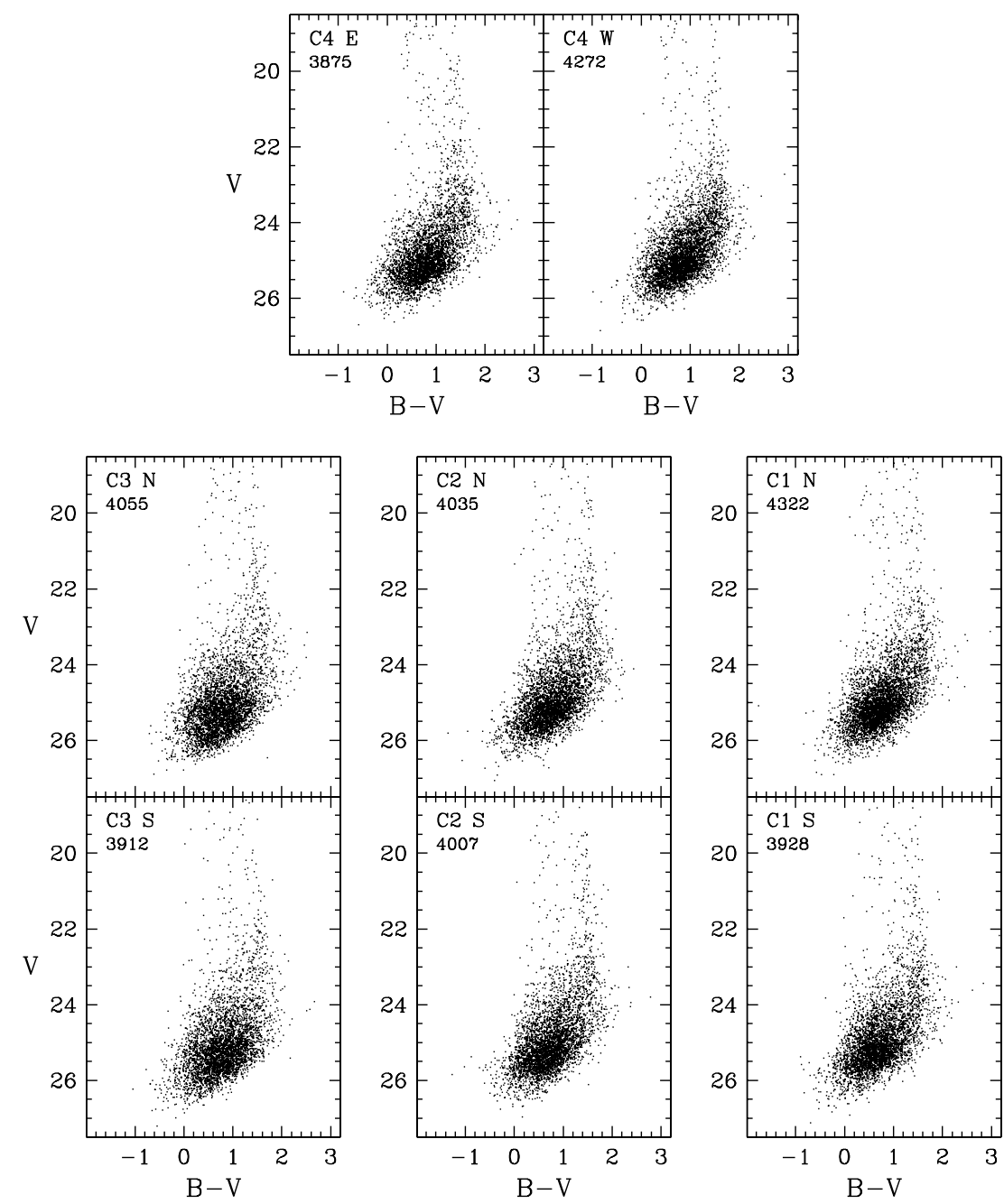

Figure 4. $V, B-V$ CMDs of field H1, from a pair of $B, V$ images with a $300 \mathrm{~s}$ exposure time; obtained in optimal observing conditions (FWHM $\sim 0$ '. $8-1{ }^{\prime \prime} 0$ ).

3, and east and west parts for CCD 4. Accordingly, CMDs corresponding to the four different CCDs of each field were labeled as follows: $\mathrm{C} 1 \mathrm{~N}$ and $\mathrm{C} 1 \mathrm{~S}$ for CCD1 the north and south parts, respectively, and similarly with CCD2 and 3, while the east and west parts of CCD4 were labeled as $\mathrm{C} 4 \mathrm{E}$ and $\mathrm{C} 4 \mathrm{~W}$, respectively. Each CCD of the LBT/LBC-blue mosaic covers about a $1.7 \times 3.9 \mathrm{kpc}^{2}$ area of M31; however, because of the trimming of the $V$ images, the CMDs corresponding to the individual CCDs in fact cover a reduced but still remarkable area roughly the size of $1.7 \times 3.4 \mathrm{kpc}^{2}$. We have accounted for this problem when dividing the CCDs and the corresponding CMDs in parts to ensure that each CMD in Figures 3 and 4 samples the same area of M31. The most striking feature in the CMDs of field S2 is a conspicuous blue plume observed in panels $\mathrm{C} 1 \mathrm{~N}, \mathrm{C} 1 \mathrm{~S}$, and $\mathrm{C} 4 \mathrm{~W}$ of Figure 3 at $V \leqslant 25.0 \mathrm{mag}$ and $B-V \leqslant 0.4 \mathrm{mag}$. This blue plume is barely discernible in $\mathrm{C} 2$ $\mathrm{N}$ and eventually disappears, moving eastward from CCD2 to CCD 3. Also intriguing is a feature seen in $\mathrm{C} 2 \mathrm{~N}$ and $\mathrm{S}, \mathrm{C} 3 \mathrm{~N}$ and $\mathrm{S}$, and $\mathrm{C} 4 \mathrm{E}$ at $V \leqslant 25.0 \mathrm{mag}$ and $0.2<B-V<0.4 \mathrm{mag}$. Finally, all of the CMDs show a variably populated, bright red plume and a sparse distribution of bright stars of intermediate colors. We believe that the blue plume is produced by young stars possibly associated with an M31 spiral arm and the galaxy disk, while the red plume is due to local M dwarfs.

The CMDs of field H1 (see Figure 4) are much less populated than those of field $\mathrm{S} 2$, and the blue plume is totally absent, which is not surprising if the blue plume in field S2 is due to the disk and spiral arm stars and if field $\mathrm{H} 1$ is instead representing the M31 halo population.

In order to correctly interpret the features we see in the CMDs in terms of the SFH and the structure of M31, a reliable evaluation of the foreground contamination due to our Galaxy is necessary. To approach this problem, we have run simulations using a well-tested star-count code for our Galaxy (see Cignoni et al. 2008; Castellani et al. 2002). In this code the MW is divided into three major Galactic components, namely, the thin disk, the thick disk, and the halo. For each of these three components an artificial population is created by a random choice of mass and age from the assumed initial mass function and star formation law, interpolating on a grid of evolutionary tracks (from the zero age main sequence to the white dwarf phase), the metallicity of which is determined by the adopted age-metallicity relation. Reddening and photometric errors of the data are convolved with magnitudes of the synthetic stars, producing a realistic CMD. The thin disk and the thick disk density laws were modeled by a double exponential with the same scale length $(3500 \mathrm{pc})$ but with a different scale height (1 kpc for the thick disk, $300 \mathrm{pc}$ for the thin disk). The halo follows a power-law decay with an exponent of 3.5 and an axis ratio of 0.8 . A local spatial density of $0.11 \mathrm{stars}^{-3}$ was adopted for the thin disk, whereas the thick disk and halo normalizations were $1 / 10$ and $1 / 500$, respectively, relative to the thin disk. 
Table 3

Range of Expected Galactic Contaminating Stars as a Function of Magnitude and Color

\begin{tabular}{lcc}
\hline \hline Magnitude Bin & Blue & Red \\
\hline $18.5 \leqslant V \leqslant 20$ & $0-1$ & $14-24$ \\
$20<V \leqslant 21$ & $0-1$ & $6-10$ \\
$21<V \leqslant 22$ & $0-1$ & $3-9$ \\
$22<V \leqslant 23$ & $0-1$ & $3-7$ \\
$23<V \leqslant 24$ & $1-3$ & $3-8$ \\
\hline
\end{tabular}

Notes. Over an area equivalent to the area covered by each of the CMDs shown in the eight panels of Figures 3 and 4. Blue: $0<B-V<0.5 \mathrm{mag}$; Red: $0.5 \leqslant B-V<1.0 \mathrm{mag}$.

The metallicity of each Galactic component was fixed at $Z=$ $0.02, Z=0.006$, and $Z=0.0002$ for the thin disk, thick disk, and halo, respectively. In order to establish quantitative limits to the Galactic star counts in field S2, all free model parameters were let to vary. In particular, the thin disk scale height was allowed to vary between 250 and $300 \mathrm{pc}$, with the thick disk and halo normalizations tested between $1 / 10$ and $1 / 20$ and between $1 / 500$ and $1 / 850$ relative to the thin disk. Table 3 summarizes the predicted star counts as a function of the magnitude and color over an area equivalent to the area covered by each of the CMDs shown in the eight panels of Figures 3 and 4. Figure 5 shows a typical simulated $\mathrm{CMD}$ for the foreground contamination in field S2, which was obtained by assuming $E(B-V)=$ $0.08 \mathrm{mag}$, and the typical internal errors of our photometry $\left(0.007<\sigma_{B}<0.296 \mathrm{mag}\right.$ and $0.008<\sigma_{V}<0.252 \mathrm{mag}$, for $20.0<V<26.0$ ). The simulation describes the contamination by Galactic stars, affecting each of the CMDs shown in the eight panels of Figures 3 and 4. This simulation demonstrates that the Galactic contamination is generally negligible at any magnitude level for $B-V \leqslant 0.4$ mag; hence, the blue plume observed in the CMDs of panels $\mathrm{C} 1 \mathrm{~N}, \mathrm{C} 1 \mathrm{~S}$, and $\mathrm{C} 4 \mathrm{~W}$ is produced by M31 stars, and it is not due to contamination by the Galactic stars. Conversely, all of the bright stars with intermediate colors are likely MW stars (of the halo and thick disk), and most of the bright red plume stars are MW thick disk M dwarfs. To make a more quantitative comparison, we have counted the number of stars (as a function of the same magnitude and color bins as in the simulation) in each of the CMDs shown in the eight panels of Figures 3 and 4 . These counts are provided in Tables 4 and 5 for fields S2 and H1, respectively. The comparison with Table 3 shows that the MW contamination clearly dominates all the CMDs of field $\mathrm{S} 2$ for magnitudes brighter than $V=21 \mathrm{mag}$, both in the blue and the red bins. In the $21<V \leqslant 22$ mag range, the MW dominates in the eastern CCDs (CCD4 E and CCD3 $\mathrm{N}$ and $\mathrm{S}$ ), but the M31 contribution increases progressively as we move westward and approach the M31 disk and, possibly, a spiral arm. Similarly, in the $22<B-V \leqslant 23$ mag bin there is an almost equal contribution of MW and M31 stars in the eastern CCDs, but M31 takes over progressively and becomes dominant in the western CCDs (CCD4 W and CCD1 N and S). Finally, M31 stars dominate all of the CMDs for magnitudes fainter than $V=23$ mag. Star counts for field H1 (see Table 5) have a smoother distribution, which is expected for a halo population. The M31 stars only dominate for magnitudes fainter than $V=$ $23 \mathrm{mag}$, while for $V<23 \mathrm{mag}$ MW and M31 stars contribute almost equally for $0.0<B-V<0.5 \mathrm{mag}$, and the MW generally dominates for $0.5 \leqslant B-V<1.0 \mathrm{mag}$.

In Figures 6 and 7 we show a $B$ image of field $S 2$ and a $B$ image of field $\mathrm{H} 1$, respectively, where we have overplotted in blue stars with $V \leqslant 25.0 \mathrm{mag}$ and $B-V \leqslant 0.2 \mathrm{mag}$, which correspond

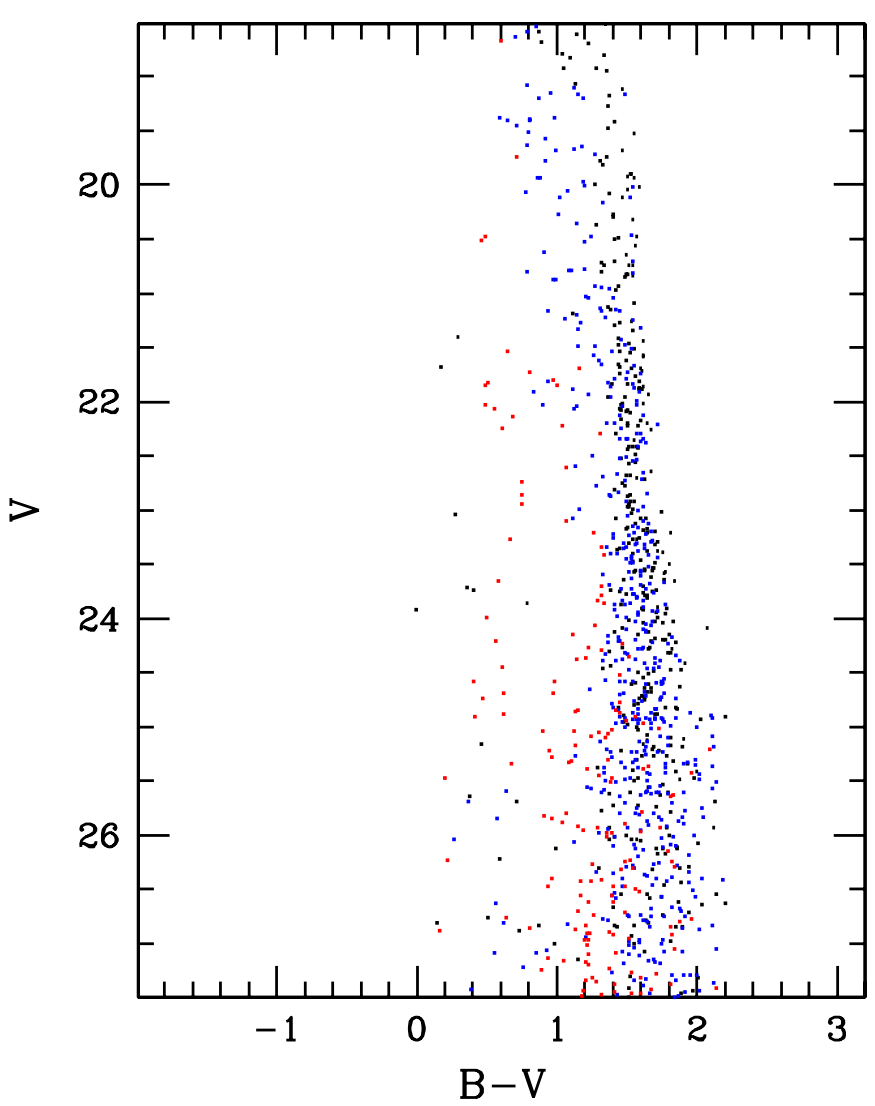

Figure 5. Foreground simulation for field $\mathrm{S} 2$, including the thin disk (black dots), the thick disk (blue dots), and the halo (red dots) stars. This CMD is on the same scale as those in Figure 3 to allow for a direct comparison.

(A color version of this figure is available in the online journal.)

to sources populating the blue plume of the CMDs, and in red stars having $V \leqslant 25.0 \mathrm{mag}$ and $0.2<B-V \leqslant 0.4 \mathrm{mag}$, which correspond to the intermediate-color features seen in Figures 3 and 4. For stars located on the upper 500 pixels of each CCD of the mosaic, we only have $B$ magnitudes because of the unfortunate trimming of the $V$ images. This is why all of these stars are missing in the CMDs of Figures 3 and 4, as well as in the images shown in Figures 6 and 7. Nevertheless, while the intermediate-color sources (red crosses) are almost homogeneously spread on all four CCDs, both in field S2 and in field $\mathrm{H} 1$, and thus likely trace the halo component, the blueplume stars (blue boxes) appear to be mainly concentrated in the upper right (northwest) part of CCD1 and in the right (west) portion of CCD4 of field S2, thus likely tracing the disk and, possibly, a spiral arm of M31. To evaluate the significance of these uneven distributions, we have counted the number of stars in the blue and intermediate plumes of each of the CMDs shown in the eight panels of Figures 3 and 4, respectively, and in the magnitude bins $V \leqslant 24.0 \mathrm{mag}$ and $24<V \leqslant 25.0 \mathrm{mag}$, separately. These counts are provided in Tables 6 and 7 for fields S2 and H1, respectively. The star counts in Table 6 show that the number of blue and intermediate-plume sources in field S2 increases dramatically, but not homogeneously, as we move westward, from CCD4 E to CCD4 $\mathrm{W}$ and from CCD3 to CCD1 and approach the M31 disk. The highest concentration of blue and intermediate-plume stars is found in CCD4 W and CCD1 $\mathrm{N}$, but it drops significantly in CCD1 S. The counts in Table 7 instead confirm the smooth stellar distribution in field $\mathrm{H} 1$, showing only a marginal increase in the number of blue and intermediate-plume stars with $24<V \leqslant 25$ mag in CCD1 
Table 4

Number of Stars in the Four CCD Mosaic of Field S2 as a Function of Magnitude and Color

\begin{tabular}{|c|c|c|c|c|c|c|}
\hline \multirow[t]{2}{*}{ Magnitude Bin } & \multicolumn{2}{|c|}{ CCD4 E } & & & \multicolumn{2}{|c|}{ CCD4 W } \\
\hline & Blue & Red & & & Blue & Red \\
\hline $18.5 \leqslant V \leqslant 20$ & 2 & 16 & & & 4 & 5 \\
\hline $20<V \leqslant 21$ & 3 & 12 & & & 1 & 11 \\
\hline $21<V \leqslant 22$ & 2 & 8 & & & 10 & 14 \\
\hline $22<V \leqslant 23$ & 4 & 13 & & & 23 & 40 \\
\hline \multirow[t]{3}{*}{$23<V \leqslant 24$} & 46 & 148 & & & 165 & 443 \\
\hline & \multicolumn{2}{|c|}{$\mathrm{CCD} 3 \mathrm{~N}$} & \multicolumn{2}{|c|}{$\mathrm{CCD} 2 \mathrm{~N}$} & \multicolumn{2}{|c|}{$\mathrm{CCD} 1 \mathrm{~N}$} \\
\hline & Blue & Red & Blue & Red & Blue & Red \\
\hline $18.5 \leqslant V \leqslant 20$ & 1 & 12 & 0 & 8 & 0 & 8 \\
\hline $20<V \leqslant 21$ & 2 & 7 & 4 & 17 & 3 & 8 \\
\hline $21<V \leqslant 22$ & 1 & 4 & 2 & 15 & 15 & 7 \\
\hline $22<V \leqslant 23$ & 3 & 13 & 4 & 23 & 17 & 36 \\
\hline \multirow[t]{3}{*}{$23<V \leqslant 24$} & 35 & 133 & 38 & 151 & 260 & 667 \\
\hline & \multicolumn{2}{|c|}{ CCD3 S } & \multicolumn{2}{|c|}{$\mathrm{CCD} 2 \mathrm{~S}$} & \multicolumn{2}{|c|}{ CCD1 S } \\
\hline & Blue & Red & Blue & Red & Blue & Red \\
\hline $18.5 \leqslant V \leqslant 20$ & 1 & 15 & 2 & 4 & 2 & 13 \\
\hline $20<V \leqslant 21$ & 1 & 7 & 6 & 10 & 2 & 8 \\
\hline $21<V \leqslant 22$ & 2 & 8 & 3 & 7 & 7 & 11 \\
\hline $22<V \leqslant 23$ & 7 & 14 & 3 & 19 & 7 & 27 \\
\hline $23<V \leqslant 24$ & 65 & 166 & 36 & 136 & 107 & 554 \\
\hline
\end{tabular}

Notes. Over an area equivalent to the area covered by each of the CMDs shown in the eight panels of Figure 3. Blue: $0<B-V<0.5$ mag; Red: $0.5 \leqslant B-V<1.0$ mag.

Table 5

Number of Stars in the Four CCD Mosaic of Field H1 as a Function of Magnitude and Color

\begin{tabular}{|c|c|c|c|c|c|c|}
\hline \multirow[t]{2}{*}{ Magnitude Bin } & \multicolumn{2}{|c|}{ CCD4 E } & & & \multicolumn{2}{|c|}{ CCD4 W } \\
\hline & Blue & Red & & & Blue & Red \\
\hline $18.5 \leqslant V \leqslant 20$ & 11 & 10 & & & 5 & $\overline{11}$ \\
\hline $20<V \leqslant 21$ & 1 & 13 & & & 3 & 6 \\
\hline $21<V \leqslant 22$ & 4 & 9 & & & 1 & 8 \\
\hline $22<V \leqslant 23$ & 3 & 9 & & & 6 & 16 \\
\hline \multirow[t]{3}{*}{$23<V \leqslant 24$} & 21 & 72 & & & 15 & 94 \\
\hline & \multicolumn{2}{|c|}{ CCD3 N } & \multicolumn{2}{|c|}{$\mathrm{CCD} 2 \mathrm{~N}$} & \multicolumn{2}{|c|}{ CCD1 N } \\
\hline & Blue & $\overline{\text { Red }}$ & Blue & Red & Blue & Red \\
\hline $18.5 \leqslant V \leqslant 20$ & 1 & 16 & 3 & 10 & 7 & 8 \\
\hline $20<V \leqslant 21$ & 3 & 3 & 4 & 6 & 3 & 13 \\
\hline $21<V \leqslant 22$ & 4 & 5 & 4 & 7 & 0 & 7 \\
\hline $22<V \leqslant 23$ & 3 & 10 & 6 & 16 & 6 & 10 \\
\hline \multirow[t]{3}{*}{$23<V \leqslant 24$} & 25 & 82 & 25 & 107 & 22 & 83 \\
\hline & \multicolumn{2}{|c|}{ CCD3 S } & \multicolumn{2}{|c|}{$\mathrm{CCD} 2 \mathrm{~S}$} & \multicolumn{2}{|c|}{ CCD1 S } \\
\hline & Blue & Red & Blue & Red & Blue & Red \\
\hline $18.5 \leqslant V \leqslant 20$ & 4 & 9 & 5 & 12 & 1 & 6 \\
\hline $20<V \leqslant 21$ & 2 & 5 & 2 & 7 & 2 & 9 \\
\hline $21<V \leqslant 22$ & 3 & 11 & 1 & 11 & 2 & 5 \\
\hline $22<V \leqslant 23$ & 5 & 10 & 6 & 10 & 6 & 12 \\
\hline $23<V \leqslant 24$ & 14 & 94 & 31 & 101 & 33 & 100 \\
\hline
\end{tabular}

Notes. Over an area equivalent to the area covered by each of the CMDs shown in the eight panels of Figure 4. Blue: $0<B-V<0.5$ mag; Red: $0.5 \leqslant B-V<1.0 \mathrm{mag}$.

$\mathrm{N}$ and CCD1 S, where the southwest corner of the halo field $\mathrm{H} 1$ perhaps touches a southeast portion of the giant stream (see Figure 2).

\section{VARIABLE STARS}

As anticipated in Section 2, the poor seeing conditions and technical problems made it rather challenging to use our data for the original purpose of studying the variable stars in these regions of M31. A crucial complication was the significant optical distortions of the LBT/LBC-blue camera (see Giallongo et al. 2008, Figure 4), particularly in the initial operation phase of LBT. We had to implement a number of different procedures and conduct several trials to detect the variable stars. Therefore, the number of variables we were able to identify is very limited 


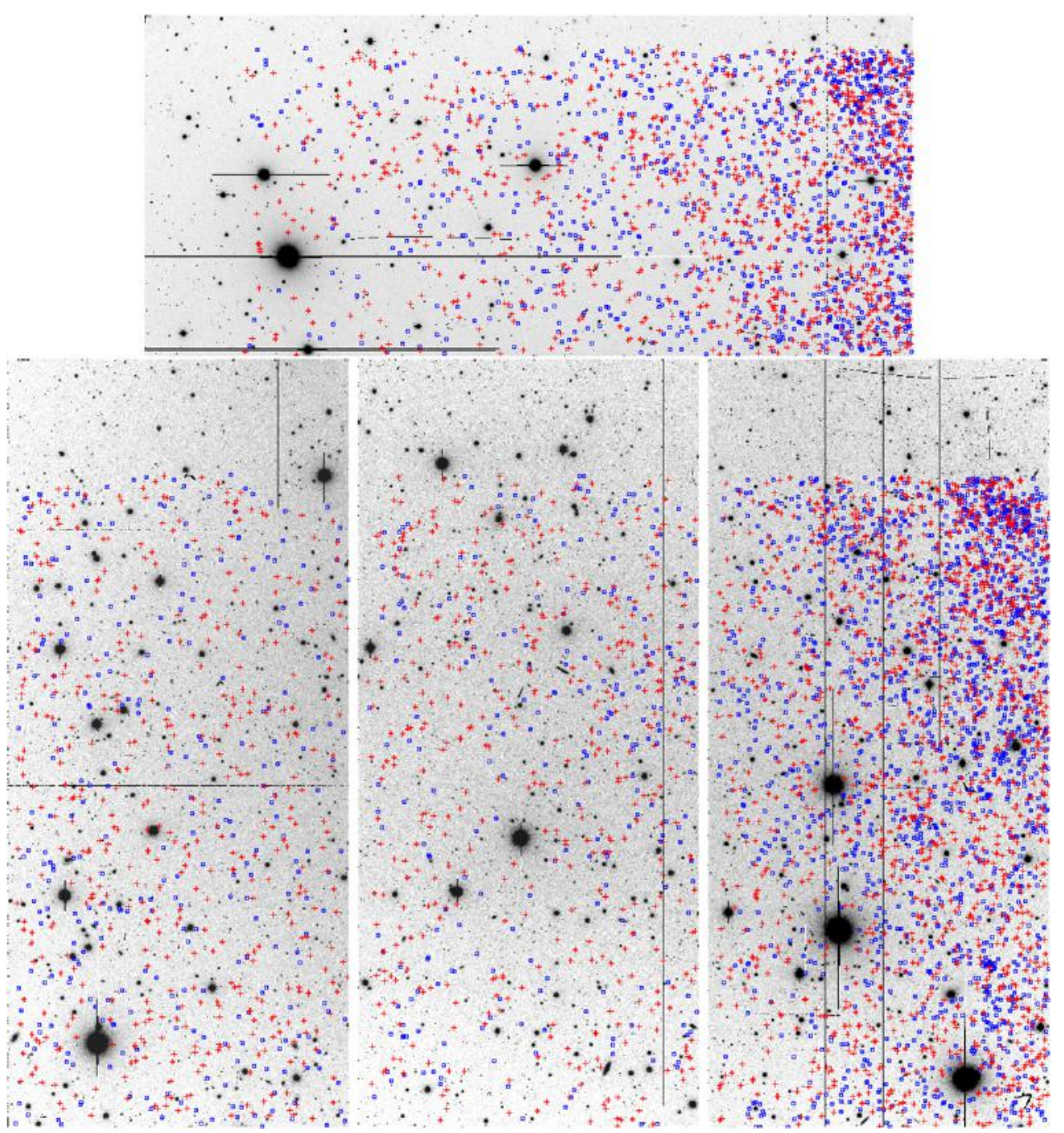

Figure 6. Position on the four CCDs mosaic of field $\mathrm{S} 2$ of stars in the blue plume of the CMD (see Figure 3). Blue boxes are stars with $V \leqslant 25.0$ and $B-V \leqslant 0.2$ mag; red crosses are stars with $V \leqslant 25.0$ and $0.2<B-V \leqslant 0.4 \mathrm{mag}$.

(A color version of this figure is available in the online journal.)

Table 6

Number of Stars in the Four CCD Mosaic of Field S2 as a Function of Magnitude and Color

\begin{tabular}{|c|c|c|c|c|c|c|}
\hline \multirow[t]{2}{*}{ Magnitude Bin } & \multicolumn{2}{|c|}{ CCD4 E } & & & \multicolumn{2}{|c|}{ CCD4 W } \\
\hline & $\mathrm{BP}$ & IP & & & $\mathrm{BP}$ & $\overline{\mathrm{IP}}$ \\
\hline$V \leqslant 24$ & 32 & 19 & & & 199 & 65 \\
\hline \multirow[t]{3}{*}{$24<V \leqslant 25$} & 139 & 195 & & & 679 & 649 \\
\hline & \multicolumn{2}{|c|}{$\mathrm{CCD} 3 \mathrm{~N}$} & \multicolumn{2}{|c|}{$\mathrm{CCD} 2 \mathrm{~N}$} & \multicolumn{2}{|c|}{ CCD1 N } \\
\hline & $\mathrm{BP}$ & IP & $\mathrm{BP}$ & IP & $\mathrm{BP}$ & IP \\
\hline$V \leqslant 24$ & 14 & 18 & 18 & 27 & 344 & 82 \\
\hline \multirow[t]{3}{*}{$24<V \leqslant 25$} & 116 & 175 & 129 & 220 & 740 & 718 \\
\hline & \multicolumn{2}{|c|}{ CCD3 S } & \multicolumn{2}{|c|}{ CCD2 S } & \multicolumn{2}{|c|}{ CCD1 S } \\
\hline & $\mathrm{BP}$ & IP & $\mathrm{BP}$ & IP & BP & IP \\
\hline$V \leqslant 24$ & 27 & 32 & 19 & 22 & 131 & 36 \\
\hline $24<V \leqslant 25$ & 142 & 220 & 83 & 166 & 469 & 544 \\
\hline
\end{tabular}

Notes. Over an area equivalent to the area covered by each of the CMDs shown in the eight panels of Figure 3. Blue plume (BP): $B-V \leqslant 0.2 \mathrm{mag}$; intermediate plume (IP): $0.2<B-V \leqslant 0.4$ mag.

if compared, for instance, to the number one would expect by extrapolating the number densities in the Brown et al. (2004) study. However, our fields are much more external than Brown et al.'s and, in fact, our number densities are in much better agreement with the number of RR Lyrae stars found by Jeffery et al. (2011) in their "halo21" field that overlaps with our field H1. This will be reviewed in further detail in Section 4.5. 


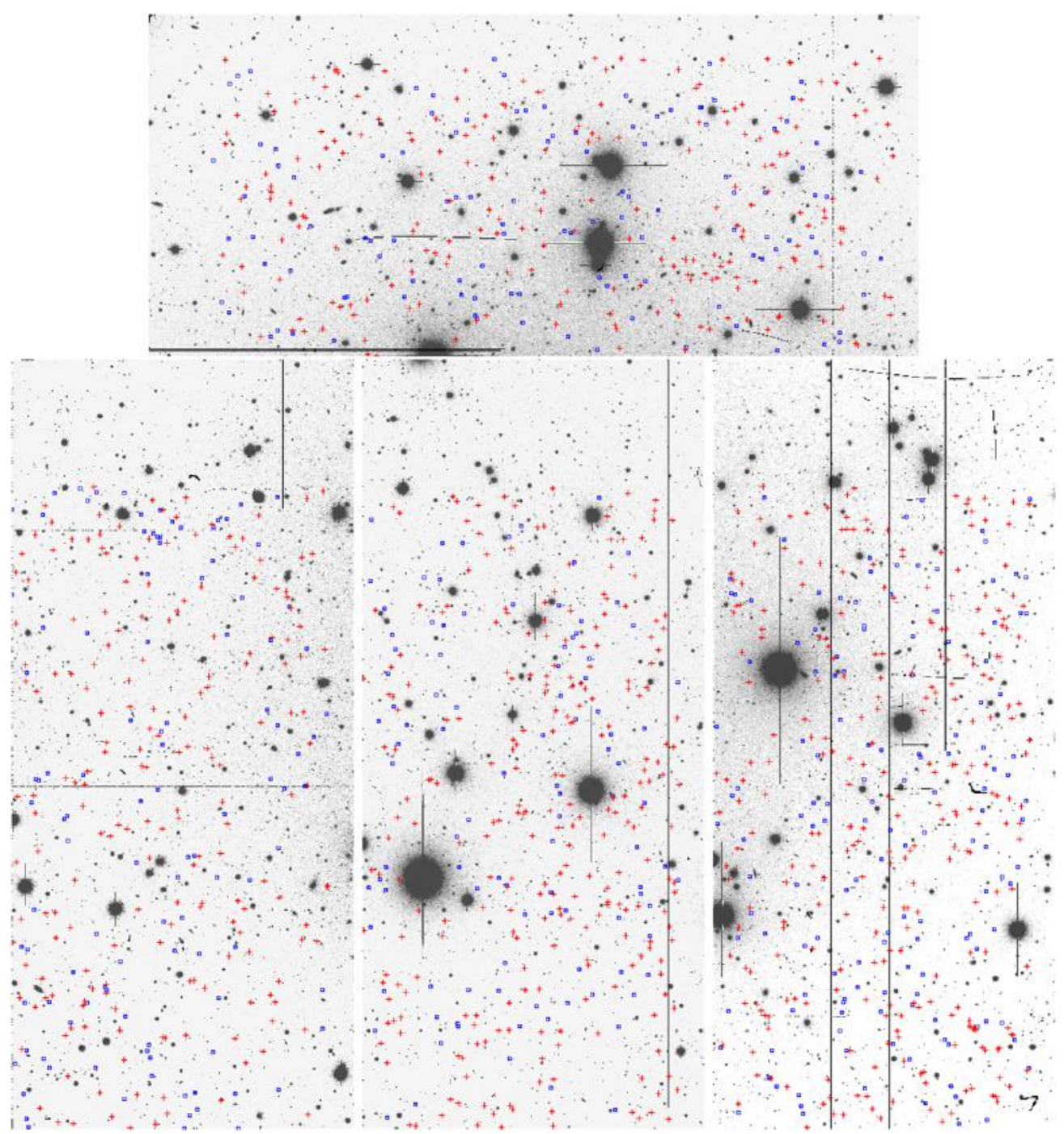

Figure 7. Position on the four CCDs mosaic of field H1 of stars in the CMD (see Figure 4) with $V \leqslant 25.0$ and $B-V \leqslant 0.2$ mag (blue boxes), and with $V \leqslant 25.0$ and $0.2<B-V \leqslant 0.4 \mathrm{mag}$ (red crosses).

(A color version of this figure is available in the online journal.)

Table 7

Number of Stars in the Four CCD Mosaic of Field H1 as a Function of Magnitude and Color

\begin{tabular}{|c|c|c|c|c|c|c|}
\hline \multirow[t]{2}{*}{ Magnitude Bin } & \multicolumn{2}{|c|}{ CCD4 E } & & & \multicolumn{2}{|c|}{$\mathrm{CCD} 4 \mathrm{~W}$} \\
\hline & $\mathrm{BP}$ & IP & & & $\mathrm{BP}$ & IP \\
\hline$V \leqslant 24$ & 8 & 14 & & & 8 & 11 \\
\hline \multirow[t]{3}{*}{$24<V \leqslant 25$} & 53 & 87 & & & 45 & 93 \\
\hline & \multicolumn{2}{|c|}{ CCD3 N } & \multicolumn{2}{|c|}{$\mathrm{CCD} 2 \mathrm{~N}$} & \multicolumn{2}{|c|}{ CCD1 N } \\
\hline & BP & IP & $\mathrm{BP}$ & IP & BP & IP \\
\hline$V \leqslant 24$ & 6 & 16 & 5 & 19 & 4 & 17 \\
\hline \multirow[t]{3}{*}{$24<V \leqslant 25$} & 46 & 82 & 45 & 93 & 57 & 109 \\
\hline & \multicolumn{2}{|c|}{$\mathrm{CCD} 3 \mathrm{~S}$} & \multicolumn{2}{|c|}{$\mathrm{CCD} 2 \mathrm{~S}$} & \multicolumn{2}{|c|}{ CCD1 S } \\
\hline & $\mathrm{BP}$ & IP & $\mathrm{BP}$ & IP & $\mathrm{BP}$ & IP \\
\hline$V \leqslant 24$ & 9 & 13 & 3 & 24 & 17 & 17 \\
\hline $24<V \leqslant 25$ & 47 & 81 & 49 & 116 & 65 & 124 \\
\hline
\end{tabular}

Notes. Over an area equivalent to the area covered by each of the CMDs shown in the eight panels of Figure 4. Blue plume (BP): $B-V \leqslant 0.2 \mathrm{mag}$; intermediate plume (IP): $0.2<B-V \leqslant 0.4$ mag. 
Table 8

Number of Bona Fide Candidate Variables Identified in Fields S2 and H1 Using the image subtraction technique, and the Numbers of Candidates Recovered in the ALLFRAME and DoPHOT Catalogs

\begin{tabular}{lcccc}
\hline \hline \multicolumn{5}{c}{ Field S2 } \\
\hline Chip & $N$ (frames) & $N$ (candidates) & $N$ (ALLFRAME) & $N$ (DoPHOT) \\
CCD 1 (upper half) & 43 & 96 & 6 & 49 \\
CCD 1 (lower half) & $\ldots$ & 2 & 0 & 2 \\
CCD 2 (total) & 43 & 143 & 40 & 74 \\
\hline \multicolumn{7}{c}{ Chip 2 (upper half) } & $N$ (frames) & $N$ (candidates) & $\ldots$ & 13 \\
CCD & 33 & 33 & $\cdots$ & \\
\hline
\end{tabular}

In the following section, we briefly describe the procedures we have implemented and the results we have obtained from the search for variable stars in CCD2 and the upper half of CCD1 of field S2, and in the upper half of CCD2 of field H1.

\subsection{Identification of the Variable Stars and Light Curves}

To identify candidate variables in our $B$ time series images of fields S2 and H1, we used the optimal image subtraction technique and the package ISIS2.1 (Alard 2000), which is known to be very efficient at identifying variables with amplitudes as low as $\Delta B<0.1 \mathrm{mag}$ in crowded fields. The package was run on the $B$ time series of CCD 1 and 2 of field S2 and CCD 2 of field $\mathrm{H} 1$. We encountered several difficulties in aligning and interpolating the images of our LBT/LBC-blue time series data with ISIS, which was likely due to the significant distortions of the LBT/LBC-blue camera. Since the regions of the LBC mosaic less affected by optical distortions are those covered by CCD2, and the best observing conditions occurred during the observations of field S2, we managed to properly align and interpolate a subset of $43 B$ images of the entire CCD2 of field S2 with ISIS, and then make the subsequent search for variable stars. Interpolation did not succeed instead for the entire CCD1; we had to divide it into two halves, and only images corresponding to the upper half of CCD1 of field S2 were successfully aligned. We encountered even more problems with the images of field $\mathrm{H} 1$, since they were generally obtained under worse seeing conditions. We divided the CCD in two parts and were only able to align and interpolate a subset of 33 images corresponding to the upper half of CCD2. After aligning and interpolating the images, we built reference images of CCD2-S2, CCD1-S2 (upper part), and CCD2-H1 (upper part). We subtracted them out from the respective time series and summed the differences of the images to obtain var.fits images, which, according to ISIS, are the maps of variable sources in the frames under study. Specifically, we used 19 and 28 frames to build two varfits images of CCD2 of field H1, 17 and 28 images for CCD2 of field S2, and 20 and 43 images for CCD1 of field S2. In order to pick up candidate variables from the var.fits images that were as faint as the RR Lyrae stars, which at minimum light in our frames were expected to have an $\mathrm{S} / \mathrm{N} \sim 2$, we had to use a very low detection threshold of 0.33 . We ended up with rather large lists of about 4000 candidate variables from each var.fits frame. Lists corresponding to the pair of varfits frames of each field were cross-correlated, thus obtaining about 2000 common candidate sources per set of images. A careful inspection of these stars returned a final catalog of 143 bona fide variables in CCD2 of field S2, 96 variables in the upper portion of CCD1 of field S2, and 33 variables in the upper portion of CCD2 of field H1. Two additional bona fide variables were also identified in the lower half of CCD1 of field S2 during a preliminary search with ISIS on the whole CCD1 of field S2. Hence, the total number of variable stars we were able to identify was 274 .

We note that many of the original candidate variables could be real variables, but we only retained those that showed periodic, unquestionable, and better sampled light curves. A summary of the total number of retained candidate variables per field, found with the above procedure, is given in Table 8. Identification (namely, ISIS ID and DoPHOT ID when available), coordinates, and a rough estimate of the period, obtained by running the Period Determination by Phase Dispersion Minimization (PDM; Stellingwerf 1978) algorithm within IRAF on the differential $B$ flux time series of these bona fide candidates is provided in Table 9 . We note that only a very small fraction of the candidates in Tables 8 and 9 have a counterpart with reliable photometry in the ALLFRAME catalogs, and hence, have a light curve on a magnitude scale, while the vast majority only have $B$-band differential flux light curves. A number of different problems caused the ALLFRAME PSF photometry of the individual phase points of the variables to be generally unreliable. These problems included crowding, particularly in the disk field (field S2); rather poor and varying seeing conditions during the observations; and technical problems with the focus and tracking of the telescope, which made the FWHM vary strongly along the frames. All of these different effects combined together so that the PSF photometry could be obtained only in a few cases and often only for the pair of frames at 0!'8 FWHM. The faintest variables were generally detected only with the image subtraction, and no "reliable" PSF photometry could be obtained for most of them with ALLFRAME; on the other hand, the brighter variables had poorly sampled light curves due to the longer periods. Even in the halo field (field H1), where variables were also searched using the Stetson variability index on the catalogs produced by the ALLFRAME reductions of CCD2, visual inspection of the images of many of the candidates showed that they often had extended PSFs caused by spikes, CCD defects, telescope tracking problems, and, in turn, unreliable photometry. In conclusion, while the present data allowed us to identify variable stars, follow-up photometry in better technical/seeing conditions will be needed to produce light curves on a magnitude scale and to fully characterize these variables. However, publishing the identification and differential flux light curves obtained in the present study will help future variability studies in these regions of M31.

The study of the light curves of a few of the bona fide candidate variables with a light curve on a magnitude scale was performed with the Graphical Analyzer of TIme Series (GRATIS), which is custom software developed at the Bologna Observatory by P. Montegriffo (see, e.g., Di Fabrizio 1999; Clementini et al. 2000). In Figure 8 we show examples of the 
Table 9

Identification and Characteristics of Candidate Variable Stars Identified in the M31 Fields S2 and H1

\begin{tabular}{|c|c|c|c|c|c|c|c|c|}
\hline \multicolumn{8}{|c|}{ CCD1 - Field $S 2$} & \multirow[b]{2}{*}{ Notes } \\
\hline $\begin{array}{l}\text { ID } \\
\text { (ISIS) }\end{array}$ & $\begin{array}{c}\mathrm{ID}^{\mathrm{a}} \\
(\mathrm{DoPHOT})\end{array}$ & $\begin{array}{c}\alpha \\
(2000)\end{array}$ & $\begin{array}{c}\delta \\
(2000)\end{array}$ & $\begin{array}{c}P \\
\text { (day) }\end{array}$ & $\begin{array}{c}B^{\mathrm{b}} \\
(\mathrm{mag})\end{array}$ & $\begin{array}{c}V^{\mathrm{b}} \\
(\mathrm{mag})\end{array}$ & Type & \\
\hline 2783 & $\ldots$ & 004845.0 & +422105 & 0.26 & $\ldots$ & $\ldots$ & RR? & $\mathrm{c}$ \\
\hline 2833 & 1576 & 004844.6 & +421944 & 0.57 & 23.64 & 23.64 & Bin.? & \\
\hline
\end{tabular}

Notes.

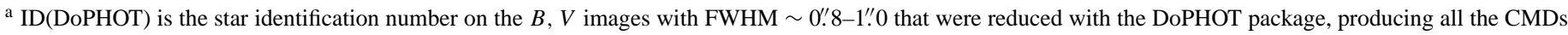
shown in the paper.

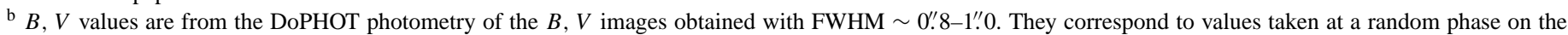

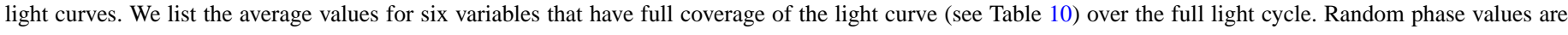
given in parentheses.

c This candidate variable falls in the portion of the $V$ frame that was trimmed during the read out of the CCDs.

d The star is on or close to a dead column of the CCD in the $B$ frame.

e The star was not measured on the 0. 8 FWHM $V$ frame because it was too bright and close to saturation.

${ }^{f}$ Variable star that has full coverage of the light curve (see Table 10).

$\mathrm{g}$ The star is close to the spike of a saturated star.

h The star is close to a dead column of the CCD in the $V$ frame.

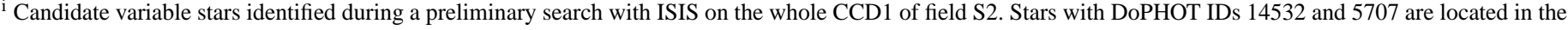
southern part of CCD1 of Field S2.

${ }^{1}$ The star is on a defect of the CCD in the $B$ frames.

$\mathrm{m}$ The star is contaminated by a bright companion.

n The star is saturated in both the $V$ and $B 0$.' 8 FWHM frames.

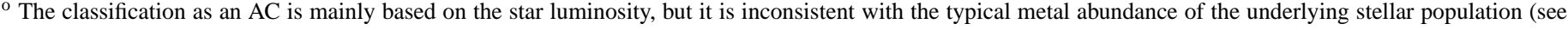
discussion in Section 4.4).

(This table is available in its entirety in a machine-readable form in the online journal. A portion is shown here for guidance regarding its form and content.)

Table 10

Identification and Properties of Confirmed Variable Stars in the M31 Field S2 with Light Curves on a Magnitude Scale

\begin{tabular}{|c|c|c|c|c|c|c|c|c|c|c|c|}
\hline Name & $\begin{array}{c}\text { ID } \\
\mathrm{b}\end{array}$ & $\begin{array}{c}\alpha \\
(2000)\end{array}$ & $\begin{array}{c}\delta \\
(2000)\end{array}$ & Type & $\begin{array}{c}P \\
\text { (days) }\end{array}$ & $\begin{array}{c}\text { Epoch }^{\mathrm{a}} \\
(-2450000)\end{array}$ & $N_{V}$ & $N_{B}$ & $\langle B\rangle^{\mathrm{c}}$ & $\begin{array}{c}A_{B} \\
(\mathrm{mag})\end{array}$ & $\langle V\rangle^{\mathrm{d}}$ \\
\hline V1 & 5089 & 004836.4 & +421457 & RRab & 0.605 & 4386.822 & 4 & 31 & 25.75 & 1.03 & 25.36 \\
\hline $\mathrm{V} 2$ & 4562 & 004838.2 & +421545 & Cepheid & 9.40 & 4385.200 & 5 & 53 & 21.48 & 0.88 & 20.62 \\
\hline V3 & 8948 & 004821.6 & +421655 & Cepheid & 3.25 & 4387.942 & 5 & 49 & 22.74 & 1.07 & 22.03 \\
\hline V4 & 6375 & 004831.8 & +421632 & Cepheid & 5.1: & 4388.400: & 5 & 51 & 22.13: & $0.84:$ & 21.47: \\
\hline V5 & $\ldots$ & 004810.2 & +421631 & Cepheid & 2.92 & 4383.700 & 5 & 51 & 22.58 & 1.29 & 21.97 \\
\hline V6 & 9171 & 004821.0 & +421939 & Binary & 0.574 & 4389.790 & 3 & 49 & 23.52 & 1.35 & 23.36 \\
\hline
\end{tabular}

Notes.

${ }^{a}$ Epochs correspond to the time of maximum light for the pulsating variables and to the time of the main minimum light for the binary system.

${ }^{\mathrm{b}}$ Identification numbers in Column 2 correspond to the ISIS IDs (see Table 9).

${ }^{c}\langle\mathrm{~B}\rangle$ values are intensity-averaged mean magnitudes.

$\mathrm{d}$ The $\langle\mathrm{V}\rangle$ values were derived by scaling from the $B$ light curves according to the procedure described at the end of Section 4.2 .

$B$ light curves of some of the variables in field S2 for which we have light curves on a magnitude scale and a reasonably complete coverage of the light cycle. They include four pulsating stars with periods of 9.4, 5.1, 3.25, and 2.92 days that we have classified as CCs on the basis of their brightness and position in the CMD (see below), an RR Lyrae star with a period of 0.605 days, and a binary system with a period of 0.574 days. The identification and properties of these six variables are provided in Table 10. Unfortunately, the PSF photometry was not good enough to obtain light curves on a magnitude scale for any of the candidate $\mathrm{ACs} / \mathrm{spCCs}$ with a period of around 1 day. $B$-band differential flux light curves for all candidate variables that we were able to identify are presented in Figures 9, 10, and 11, which are published in their entirety in the online journal.

\subsection{Classification of the Candidate Variables}

Since we only have differential flux light curves for the vast majority of the candidate variables in Table 9, we do not have information on their magnitude and on the amplitude of their light variation. This complicates the identification of the type of variability, since the only characteristic parameters we can use to classify the variables are the preliminary period and the shape of the light curve.

The candidate variables have periods in the range of 0.12 to 9.4 days. Thus, although our observing strategy was mainly devised to optimize the detection of RR Lyrae stars, it also turned out to be adequate to identify longer period variables. According to the range in the period spanned by the candidate variables, our sample is likely to contain: RR Lyrae stars $(0.2<$ $P<1$ days), Anomalous $(0.3<P<2.5$ days $)$ and Population II $(P<10$ days $)$ Cepheids, and short- and intermediate-period CCs $(1<P<10$ days $)$. For 138 candidate variables we also have an indication of magnitude because were measured on the pair of $B, V$ images of field $\mathrm{S} 2$ and $\mathrm{H} 1$ with an FWHM $\sim 0^{\prime \prime} 8$ and thus have $B, V$ magnitudes from the DoPHOT photometry (see Table 8). Although the DoPHOT magnitudes for the variables correspond to values at the random phase on the light curves, they allow us to place the candidates on the 


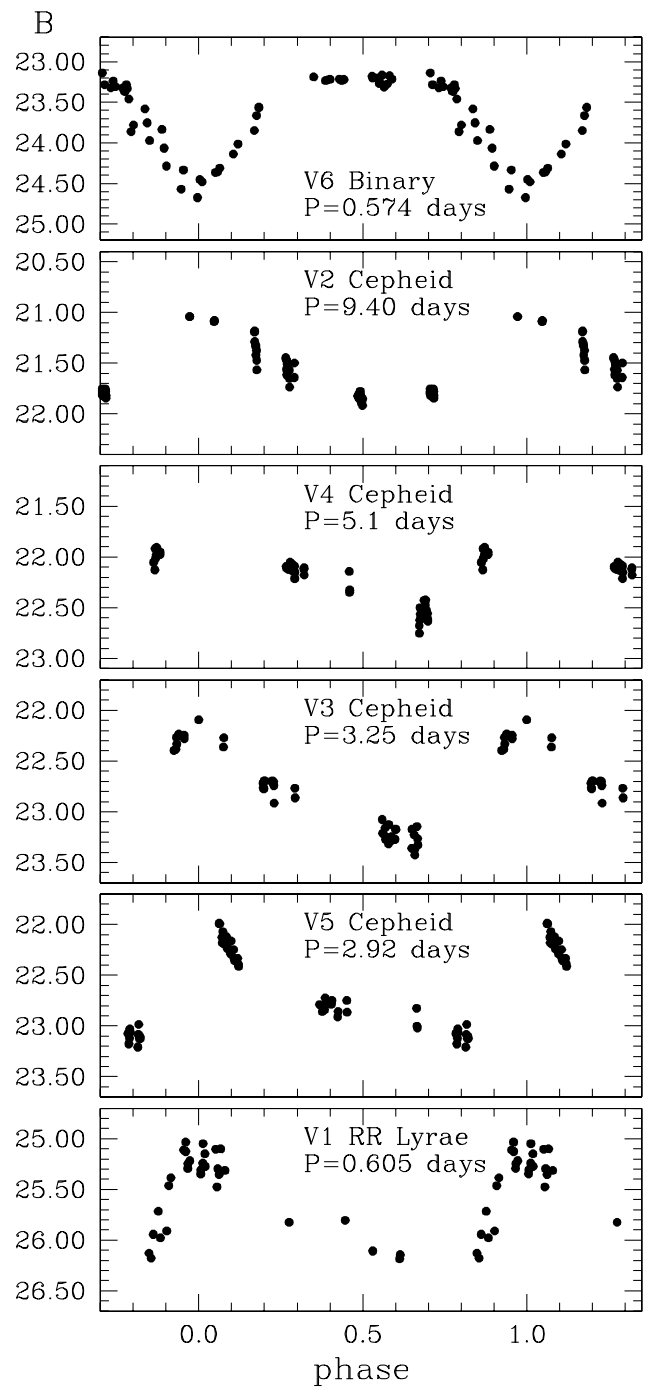

Figure 8. Examples of $B$ light curves for four Cepheids, an RR Lyrae star, and a binary system detected in the CCD1 of field S2. Each data point corresponds to a $300 \mathrm{~s}$ exposure. Typical error bars of the individual data points are in the range of 0.01 to $0.02 \mathrm{mag}$ for the CCs, $0.11-0.17 \mathrm{mag}$ for the candidate Anomalous/short-period Cepheids, and from 0.13 to 0.38 mag for the RR Lyrae stars.

CMDs (see Figures 12, 13, and 14) and thus give us some hints about their type of variability. The location on the CMDs and the periodicities of the variables at $V \sim 25-25.4 \mathrm{mag}$ confirm that they likely are RR Lyrae stars tracing the HB of the M31 old stellar component and, perhaps, Population II Cepheids (although the tentative periods generally below 1 day make a P2C classification unlikely), while variables having $V \leqslant 24$ mag are likely short- and intermediate-period CCs. On the other hand, the classification of the candidates located more than 1 mag above the $\mathrm{HB}$ at $V$ in the range of 23.5 to 24.5 mag is not easy since the luminosity would suggest that they are ACs, while the periods, which are generally well below 1 day, would make them more likely to be RR Lyrae stars. However, the AC hypothesis does not seem consistent with the typical metal abundance of the stellar population in these M31 fields, but, if these candidates are RR Lyrae stars, their brightness appears to be inconsistent (i.e., too bright) with the luminosity of the stars at the red giant branch tip, unless these variables are contaminated (i.e., blended) by the other stars. In this respect, it is interesting that no such intermediate luminosity candidates were detected in field $\mathrm{H} 1$, which is definitely less crowded than field S2. This point will be discussed in more detail in Section 4.4. To classify the candidate variable stars we have combined the information on the period, shape of the light curve, and position on the CMD (when available). We also visually inspected the $B, V$ images with the FWHM $\sim 0^{\prime \prime} .8$ at the position of each candidate variable detected by ISIS, thus revealing the saturated sources, CCD defects, and other problems (see notes of Table 9), as well as objects too faint to be reliably measured with DoPHOT, which could still be tentatively classified. The shape of the light curve also revealed several eclipsing binary systems (see Figures 9, 10, and 11), among which a number of detached systems are certainly worthy of further investigation. The variability types deduced from this procedure are provided in Column 8 of Table 9, where uncertain periods or type classifications have been flagged with a question mark. Our sample includes 96 bona fide and 31 candidate RR Lyraes, 54 bona fide and 17 candidate Cepheids (classical, anomalous, or short period), 14 bona fide and 2 candidate binary systems. For the remaining 60 variables no unambiguous classification was possible. However, the unclassified objects are likely to include a number of mainsequence variables (see, e.g., Baldacci et al. 2005), such as $\beta$ Cepheids $(P<0.3$ days $)$ and Be stars $(0.4<P<3$ days $)$, populating the blue plume at $B-V \sim 0.0 \mathrm{mag}$.

Figures 12, 13, and 14 show the CMDs of the upper part of CCD1 of field S2, the whole CCD2 of field S2, and the upper part of CCD2 of field $\mathrm{H} 1$, respectively. The candidate variables are plotted as large filled circles, and we have used different colors for the different types of variability. In the figures, the long-dashed lines around $V=25.2 \mathrm{mag}$ show the boundaries of the theoretical IS for the RR Lyrae stars (Di Criscienzo et al. 2004), and of those around $V=24.5 \mathrm{mag}$ the boundaries of the IS of ACs with $Z=0.0004$ and $1.3<M<2.2 M_{\odot}$ (Marconi et al. 2004). This is the highest metallicity allowed for ACs. ${ }^{17}$ The dotted heavy lines instead represent the first overtone and fundamental blue edges (blue lines) and the fundamental red edge (red line) for $\mathrm{CC}$ models with $Z=$ $0.008, Y=0.25$ and $3.25<M / M_{\odot}<11$ (Bono et al. $1999,2002)$. To plot the theoretical IS boundaries on the CMDs we have adopted $E(B-V)=0.08 \mathrm{mag}$, which was obtained by interpolating on the Schlegel et al. (1998) maps, $A_{V}=3.315 E(B-V)$ and $A_{B}=4.315 E(B-V)$ from Schlegel et al. (1998), and $\mu_{0}(\mathrm{M} 31)=24.43 \mathrm{mag}$. The latter value was obtained by correcting the distance modulus measured by McConnachie et al. (2005) from the M31 red giant branch tip for $E(B-V)=0.06 \mathrm{mag}$ and $A_{I}=1.94 E(B-V)$ (Schlegel et al. 1998) to our adopted reddening of $E(B-V)=0.08$ mag.

It should be noted that these variables are plotted in the CMDs using magnitudes and colors sampling random phases of the $B$ and $V$ light curves because we generally have only a few measurements of magnitude for the variables, and in many cases, we only have the pair of $B, V$ magnitudes that correspond to the two best images used to build the CMDs. They span a very large range in color and fall well beyond the boundaries of

\footnotetext{
17 As reviewed by Caputo (1998) for low-metal abundances $(Z \leqslant 0.0004)$ and relatively young ages ( $\leqslant 4 \mathrm{Gyr}$ ), the effective temperature of Zero-age horizontal branch (ZAHB) models reaches a minimum $\left(\log T_{\mathrm{e}} \sim 3.76\right)$ for a mass of about 1.0-1.2 $M_{\odot}$, while if the mass increases above this value, both the luminosity and the effective temperature start increasing, forming the so-called ZAHB turnover from which ACs are expected to evolve. For larger metallicities, the more massive ZAHB structures have brighter luminosities but effective temperatures rather close to the minimum effective temperature, so that ACs are not predicted. Observationally, ACs are mainly detected in the very metal poor dSphs and rarely in GCs.
} 

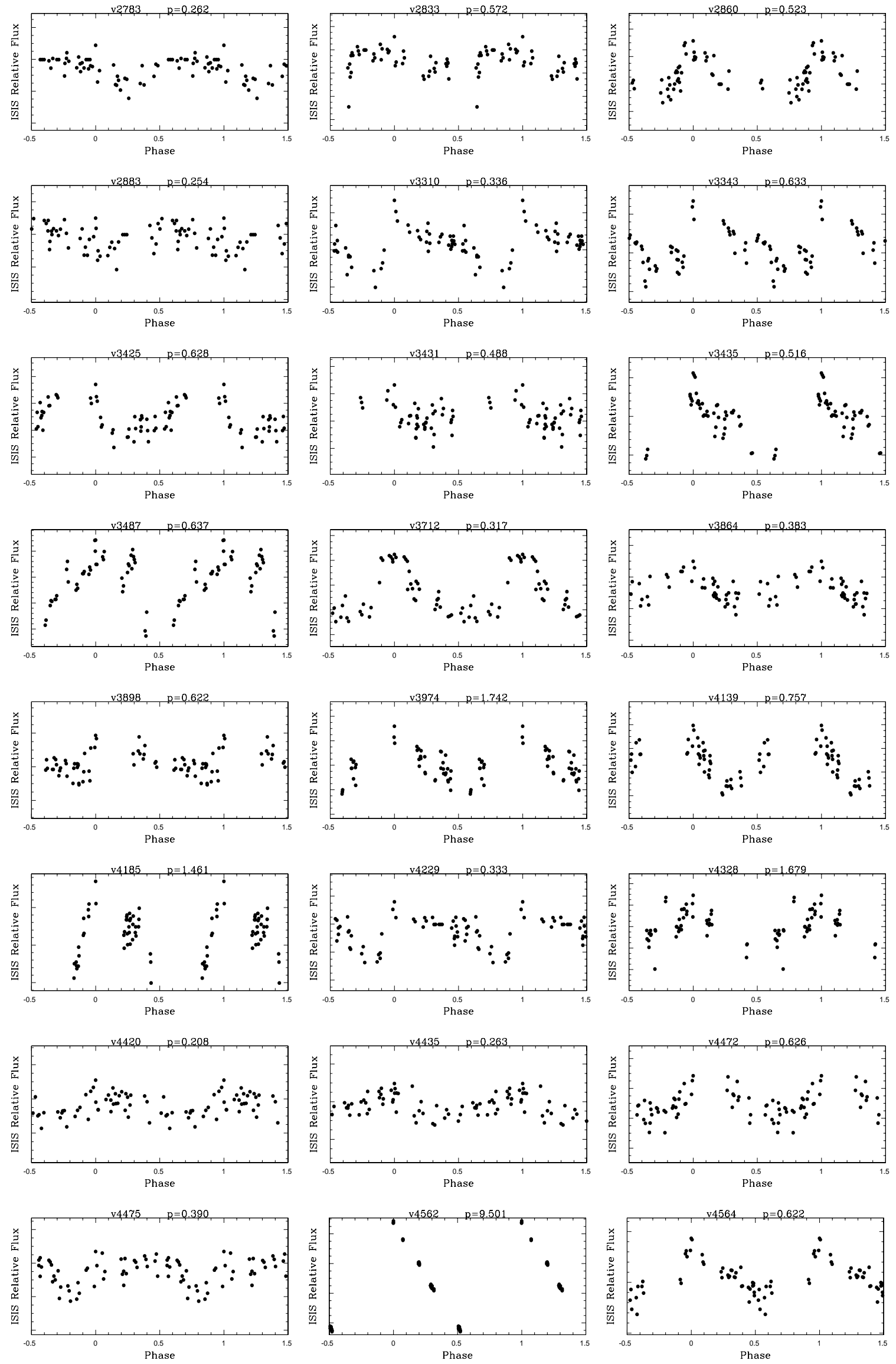

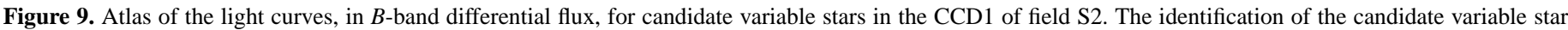

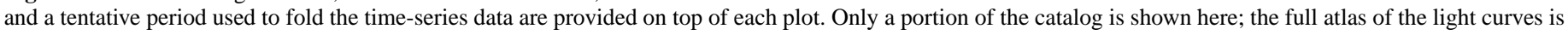
published in the online journal.

(An extended version of this figure is available in the online journal.) 

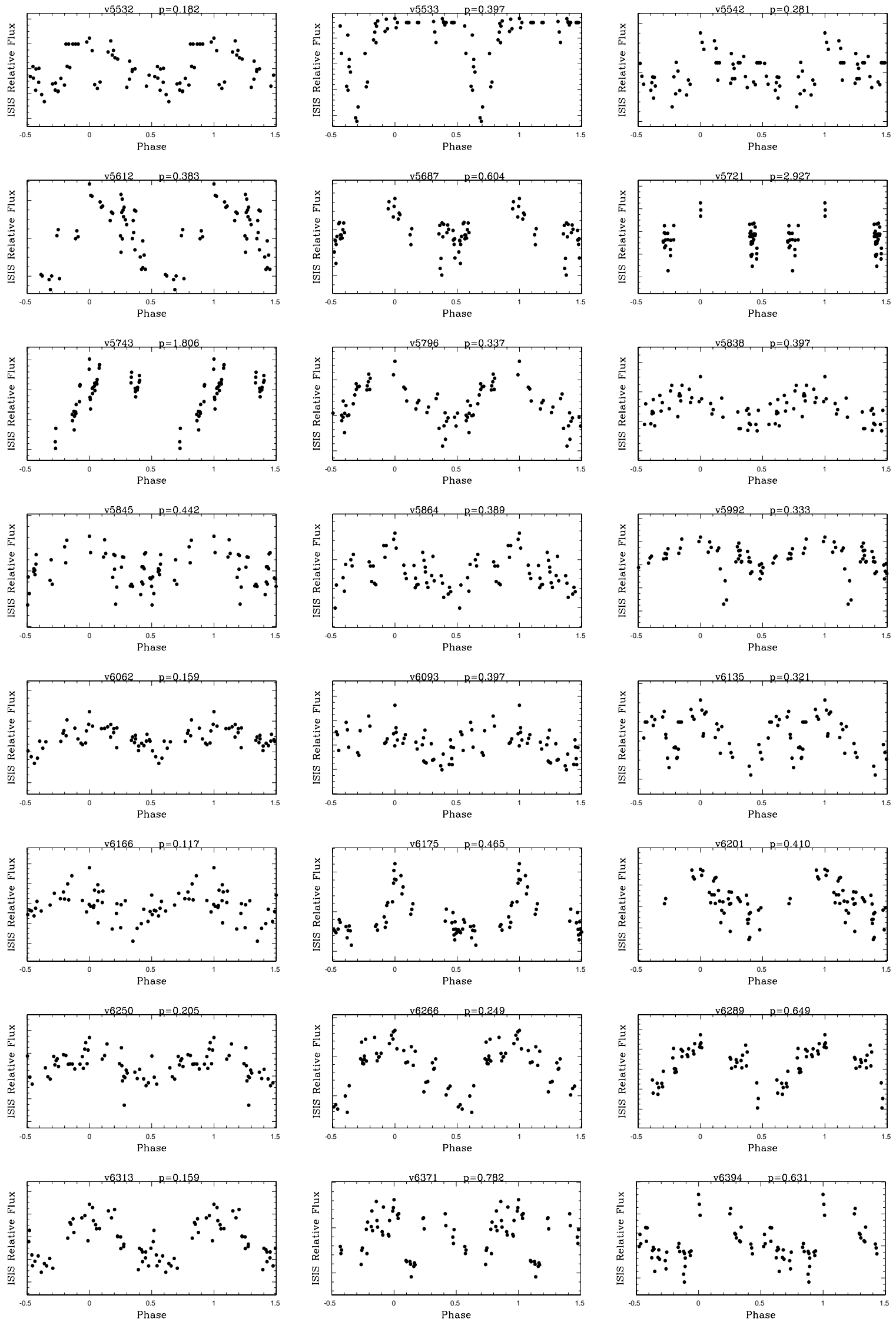

Figure 10. Same as Figure 9, except for candidate variable stars in the CCD2 of field S2.

(An extended version of this figure is available in the online journal.) 

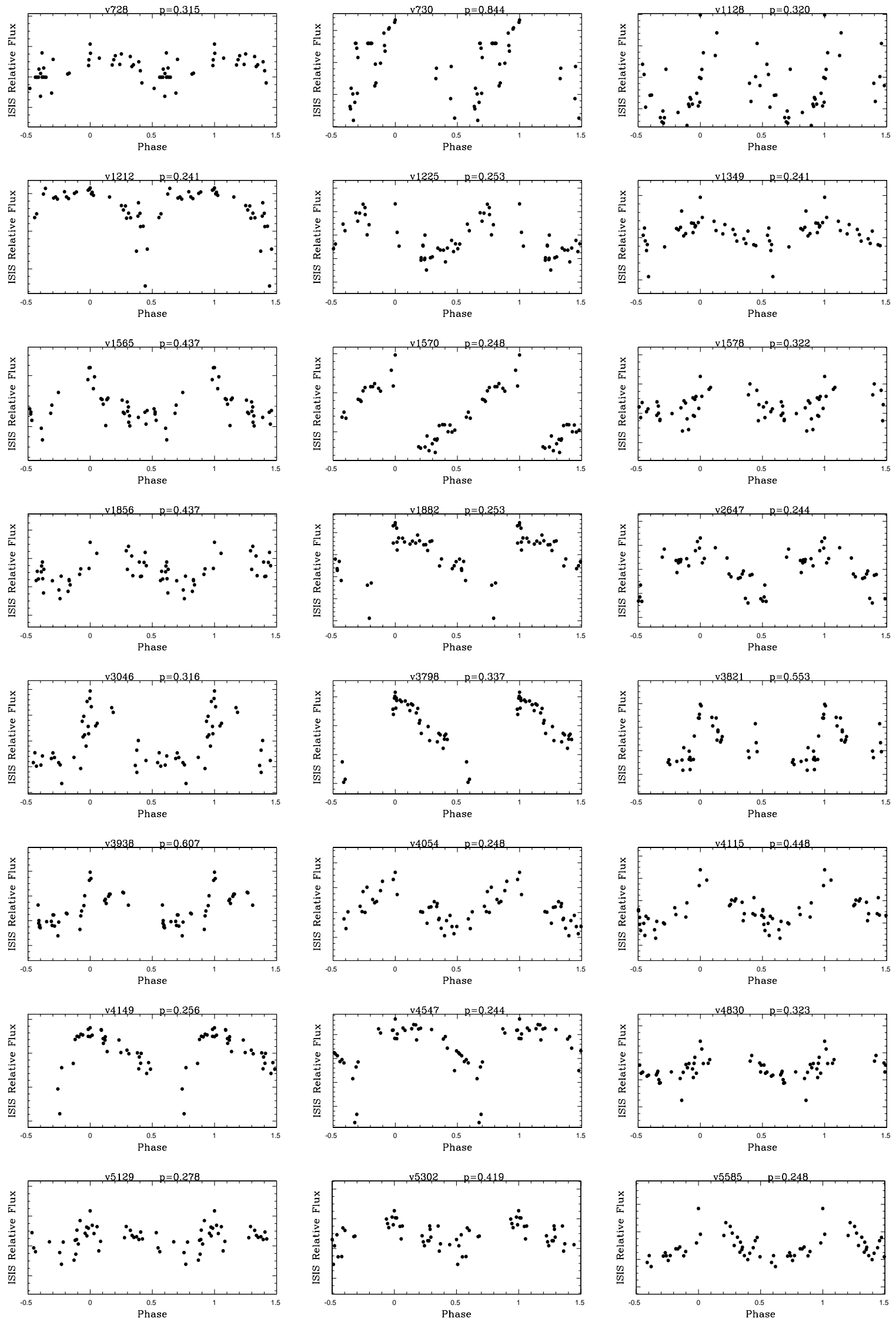

Figure 11. Same as Figures 9 and 10, except for candidate variable stars in the CCD2 of field H1.

(An extended version of this figure is available in the online journal.) 


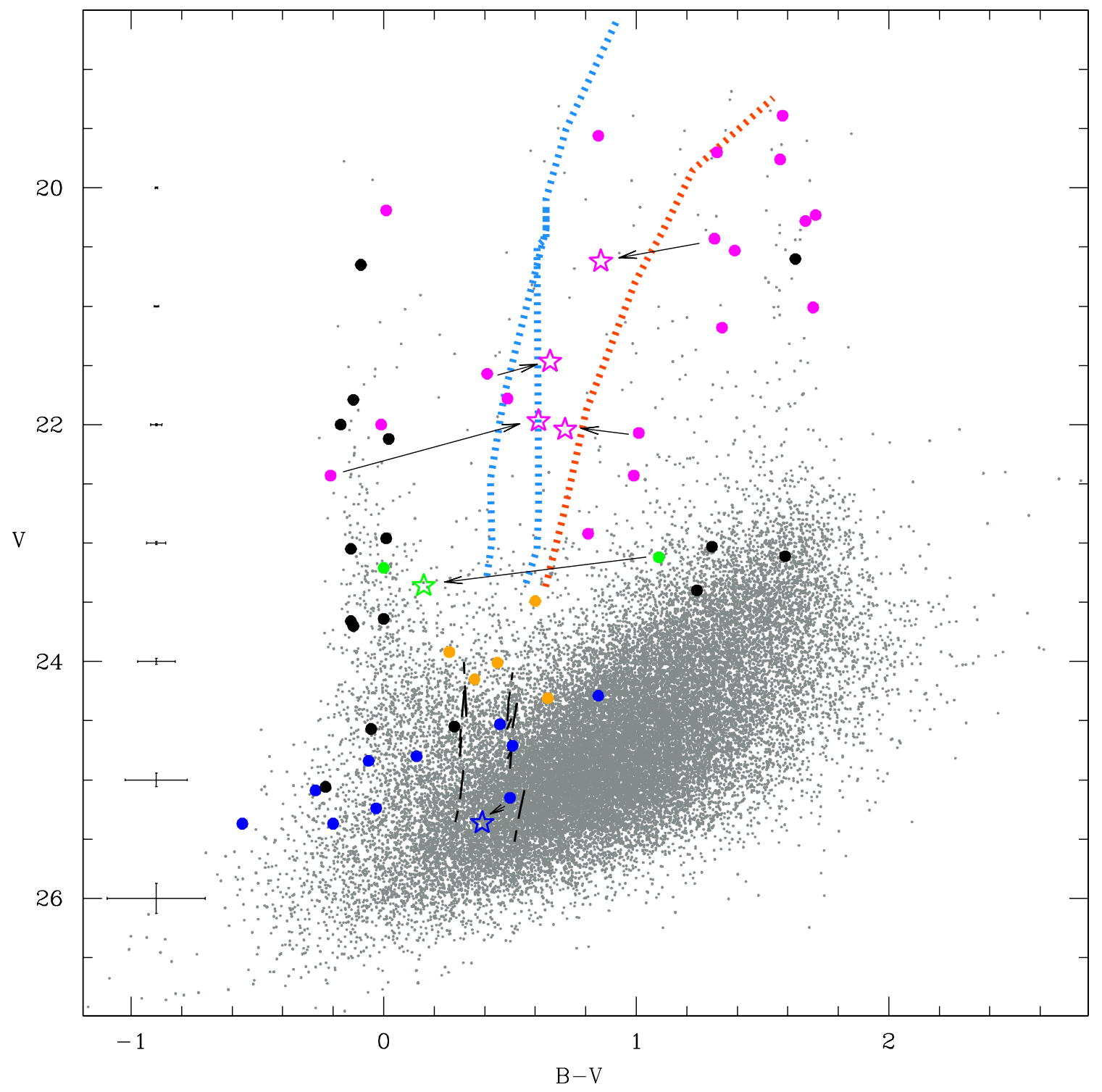

Figure 12. The $V, B-V$ CMD of the region of field $\mathrm{S} 2$ imaged on the upper portion of the CCD1, with different types of variable stars plotted by large filled dots; they include the RR Lyrae stars (around $V \sim 25-25.4 \mathrm{mag}$, blue dots), the candidate ACs/spCCs (around $V \sim 24 \mathrm{mag}$, orange dots), the CCs ( $V \leqslant 23 \mathrm{mag}$, magenta dots), the binary systems (green dots), and the candidate variables with uncertain classification (black dots). All of these variables are plotted according to their random phase magnitudes and colors. Large open stars mark five pulsating variables (blue and magenta stars) and one binary system (green star) for which we have a good sampling of the $B$ light curves (see Figure 8); their magnitudes and colors correspond to the average values along the full light cycle. Arrows connect the random phase values of these six variables to the mean values over the full light cycle. The long-dashed black lines show the boundaries of the theoretical ISs for the RR Lyrae stars and for the ACs with $Z=0.0004$ and $1.3<M<2.2 M_{\odot}$ (Marconi et al. 2004). The dotted heavy lines represent the first overtone and the fundamental blue edges (blue lines) and the fundamental red edge (red line) for the CC models with $Y=0.25$ and $3.25<M / M_{\odot}<11$ and $Z=0.008$ (Bono et al. 1999, 2002).

(A color version of this figure is available in the online journal.)

the ISs because of the decoupling of their $B, V$ magnitudes. The six variables listed in Table 10 have a better sampling of their light curves in magnitude scale, and hence they are also plotted in Figure 12 using the $\langle V\rangle$ magnitudes and $\langle B\rangle-\langle V\rangle$ colors we obtained by averaging over the full light cycle (large open stars), and with arrows connecting the average values to the random phase DoPHOT magnitudes. When the average values are used, the Cepheids and RR Lyrae stars populate the rather narrow region of the CMD that corresponds to the classical IS. This exercise illustrates how much the candidate variables plotted at the random phase could move in the $V, B-V$ plane, and it also demonstrates the detection potential and analysis capabilities of our study, which is the main purpose of the present paper.

We also note that, although the number of $B$ phase points would, in principle, be adequate to obtain a reliable estimate of the $B$ amplitude and average magnitude of the variable stars, coverage of the $V$-band light curve is very sparse (only 3-8 phase points in the best cases; see Table 1). To recover the average magnitudes in the visual band, and thus be able to plot correctly the six variable stars on the CMDs, we used the star's $B$-band light curve as a template, and we properly scaled it in amplitude to fit the few available $V$ data points. To constrain the scaling factor for the RR Lyrae stars we used amplitude ratios $A(B) / A(V)$ computed using literature $B, V$ light curves of the RR Lyrae stars with good light-curve parameters. These were selected from a number of Galactic globular clusters (GGCs; see Di Criscienzo et al. 2011 for details), and we used amplitude ratios and phase lags taken from Freedman (1988) and Wisniewski \& Johnson (1968) for the Cepheids. Finally, we used an amplitude ratio of 1 for the binary system, 


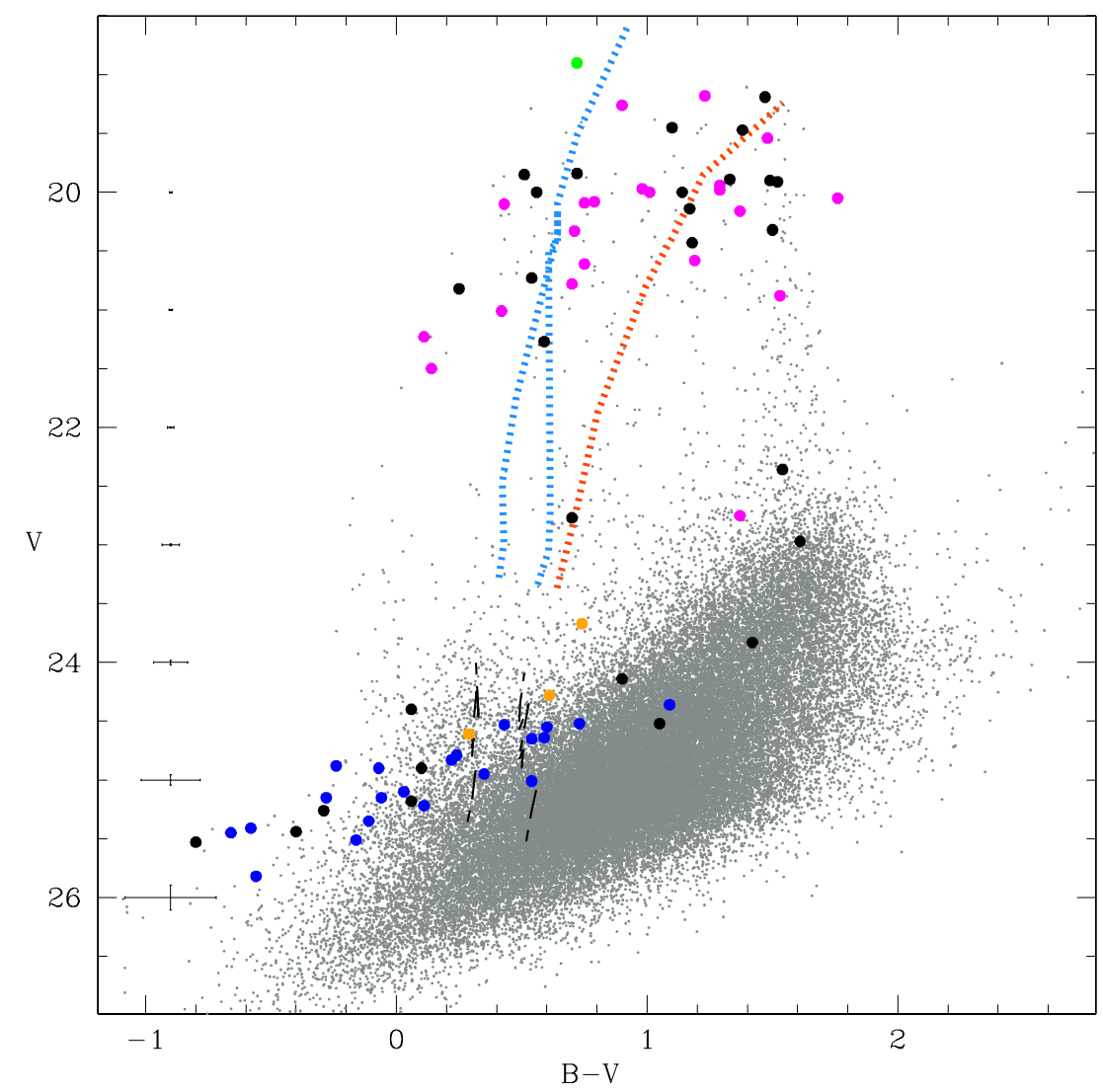

Figure 13. Same as Figure 12, except for variable stars detected in the whole CCD2 of field S2. (A color version of this figure is available in the online journal.)

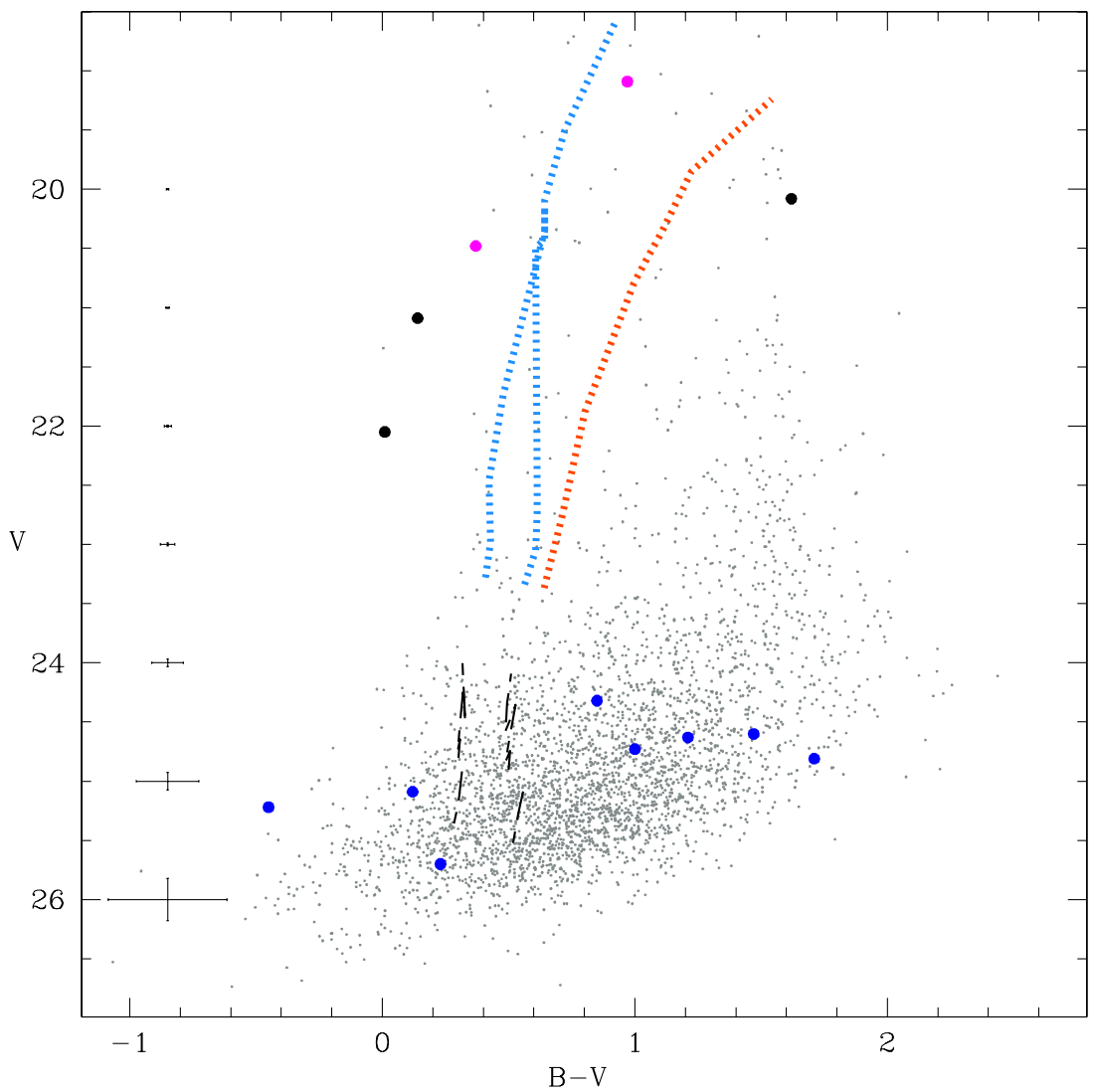

Figure 14. Same as Figure 12, except for variable stars detected in the upper portion of the CCD2 of field H1. (A color version of this figure is available in the online journal.) 
Table 11

Number of Bona Fide Variables Divided by Type and Plotted in the CMDs in Figures 12, 13, and 14

\begin{tabular}{|c|c|c|c|c|}
\hline \multicolumn{5}{|c|}{ Field S2 } \\
\hline Chip & RR Lyrae Stars & $\mathrm{CCs}$ & Bin. & Uncertain Type \\
\hline CCD 1 (upper half) & 9 & 18 & 2 & 20 \\
\hline CCD 1 (lower half) & 1 & 0 & 0 & 1] \\
\hline CCD 2 (upper half) & 10 & 14 & 1 & 10 \\
\hline CCD 2 (lower half) & 11 & 7 & 0 & 21 \\
\hline \multicolumn{5}{|c|}{ Field H1 } \\
\hline Chip & RR Lyrae Stars & $\mathrm{CCs}$ & Bin. & Uncertain Type \\
\hline CCD 2 (upper half) & 8 & 2 & 0 & 3 \\
\hline \multicolumn{5}{|c|}{ Total Numbers } \\
\hline & RR Lyrae Stars & $\mathrm{CCs}$ & Bin. & Uncertain Type \\
\hline Total & 39 & 41 & 3 & 55 \\
\hline
\end{tabular}

since we do not expect to see significant chromatic effects for binaries.

Given that the RR Lyrae stars are at the faint limit of our photometry ( $V \sim 25.5 \mathrm{mag}$ ), where the completeness drops off rapidly, understanding the completeness of our study would be important. Our data clearly suffer from a number of shortcomings that hamper a traditional analysis, making it difficult to estimate the fraction of variables in each class. Fortunately, our field H1 overlaps with Jeffery et al.'s (2011) field "halo21," thus allowing a direct comparison of the number of RR Lyrae stars found by the two studies and allowing us to draw some conclusions on the completeness of our variable star detections. Jeffery et al. (2011) found three RR Lyrae stars in their 3.5 × 3.7 HST/ACS field "halo21," which corresponds to a number density of $0.23 \mathrm{RR}$ Lyrae $\operatorname{arcmin}^{-2}$. According to Table 9, we have found 15 bona fide RR Lyrae stars in the $7.2 \times 8.6 \operatorname{arcmin}^{2}$ upper portion of field H1 that we analyzed for variability, corresponding to a number density of $0.24 \mathrm{RR}$ Lyrae arcmin $^{-2}$. The two numbers compare very favorably, thus showing that, on the assumption that the number density of the RR Lyrae stars does not vary significantly through field H1, we have attained a good completeness of the RR Lyrae detections in this field.

However, our field S2 is too far away from Jeffery et al.'s "disk" field to make a similar comparison meaningful.

\subsection{Spatial Distribution of the Variable Stars}

Out of the total sample of 274 bona fide candidate variables identified in our study, we have a magnitude estimate for 138 stars. They include 83 objects with firm classifications and 55 objects of uncertain types. Among the latter are eight variables with typical magnitudes of ACs that will be discussed in more detail in Section 4.4. The subdivision in types of these 138 stars is summarized in Table 11. In order to check whether the spatial distribution of the different types of variables can provide some hints to the underlying parent stellar population, we have also subdivided the variables into the various subfields where they are located.

Discarding the lower half of CCD1 of field S2 where the identification of variables with the image subtraction failed, and with the caveat that our statistics can, in general, be incomplete, it is interesting to note that the number of RR Lyrae stars remains almost constant in the four subfields that we have analyzed with ISIS, while the number of CCs decreases dramatically in field H1 where only two CCs were detected. This is consistent with the RR Lyrae stars tracing the M31 halo and with field H1 being a typical halo field of M31. Furthermore, the number of CCs also drops significantly as we move from north (the upper half of CCD1) to southeast (the lower half of CCD2), moving away from the disk of M31. The highest concentration of CCs is found in the upper part of CCD1 of field S2, suggesting that this region may be crossed by a spiral arm of M31 that also produces the blue plume in the CMD and the candidate main-sequence variables (namely, Be and $\beta$ Cepheid stars). Finally, the eight supposed ACs are all found in field S2, while field $\mathrm{H} 1$ does not seem to contain any candidate variables at $V \sim 24$ mag.

\subsection{Stellar Populations of Different Age and Chemical Composition}

The differences in stellar population shown by the CMDs of fields $\mathrm{S} 2$ and $\mathrm{H} 1$ are consistent with the different types of variable stars found in these regions of M31 and, as noted in Section 4.3, confirm that H1 is a typical halo field. The small number of variables detected in CCD2 of field $\mathrm{H} 1$ and the lack of variables in the range of $V \sim 23$ to 24 mag are consistent with the rather peripheral location and low stellar density of this field.

Similarly, both the appearance of the CMD and the properties of the variables in the various portions of field S2 sampled, respectively, by the whole CCD2 and the upper part of CCD1 reveal significant differences and confirm the complexity of the stellar population in this region of M31. With reference to Figures 12 and 13, the portion of field S2 imaged on CCD2 not only lacks the CMD blue plume and the main-sequence bright variables, but it also has a few candidate variables at intermediate luminosity ( $V \sim 22-24 \mathrm{mag}$ ). Specifically, in the whole CCD2 of field S2, only 6 candidate variables are found in the magnitude range of $V \sim 24$ to $V \sim 22 \mathrm{mag}$, while 19 such variables are present in the upper portion of CCD1, corresponding to only one-half of the area of field S2 covered by CCD2. Furthermore, almost all of the bright variables in CCD2 of field S2 have $V \leqslant 21.5 \mathrm{mag}$. A large fraction of them is found within the classical IS, while the bright candidate variables in CCD1 of field S2 fall, on average, outside of the strip with their random phase magnitudes. We note that the three variables marked by orange circles in Figure 13, which could either be ACs/spCCs or bright RR Lyrae stars, are all located in the lower part of CCD2.

To get hints on the age and metal abundance of the composite stellar population in field S2, we have compared the CMD of the upper part of CCD1 of field S2 (Figure 12) with the isochrones of Girardi et al. (2002) for ages in the range of 63 to $708 \mathrm{Myr}$ and metal abundances of $Z=0.008$ (Figure 15) and $Z=0.019$ (Figure 16), respectively, to fit the young stellar component 


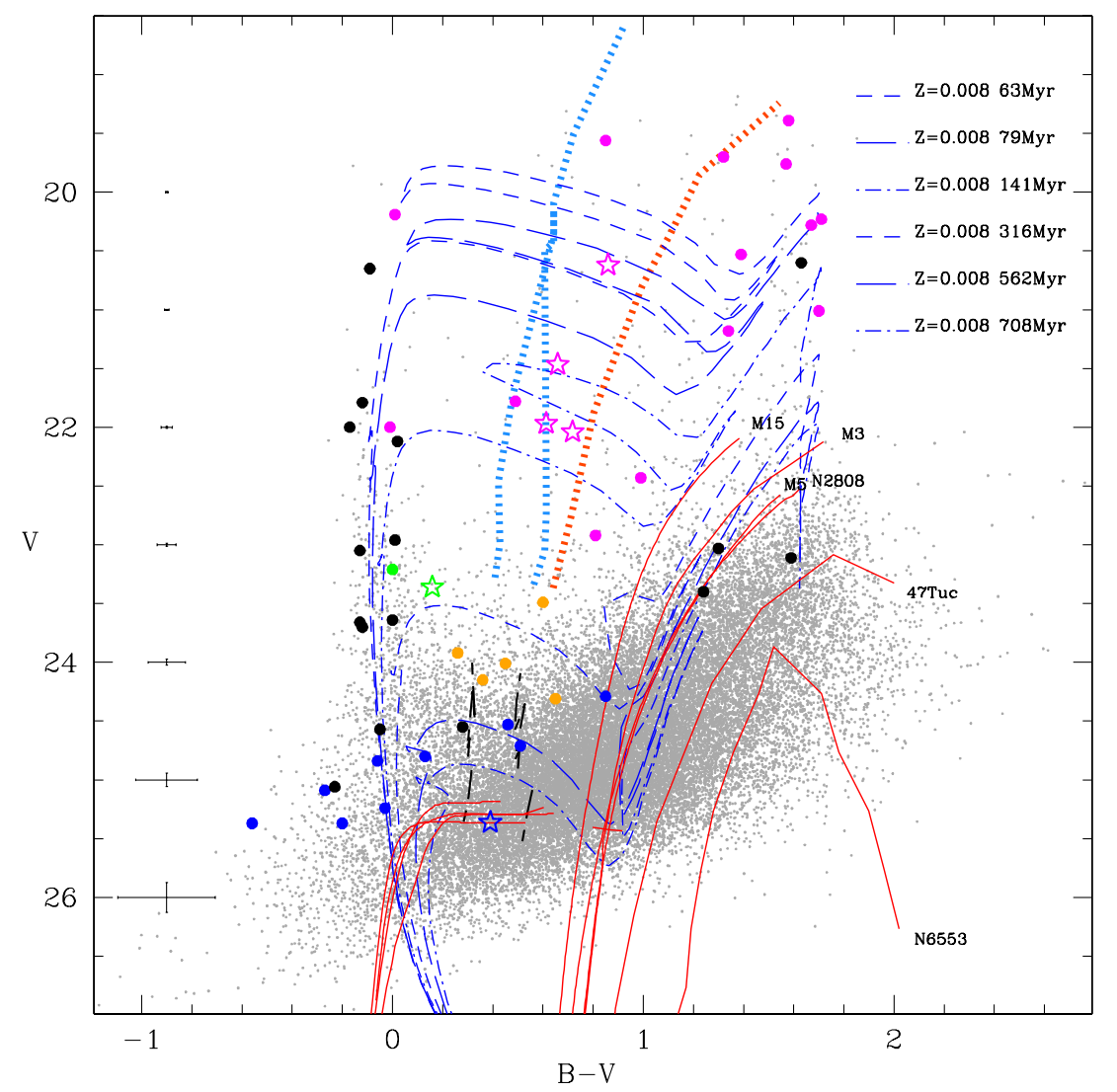

Figure 15. Same as Figure 12 with the following superimposed: 1) isochrones from Girardi et al. (2002) for a metal content of $Z=0.008$ and ages of $63,79,141,316$, 562, and $708 \mathrm{Myr}$ (blue lines); and 2) the mean ridge lines of the GGCs M15, M3, M5, NGC 2808, 47 Tuc, and NGC 6553 (red lines).

(A color version of this figure is available in the online journal.)

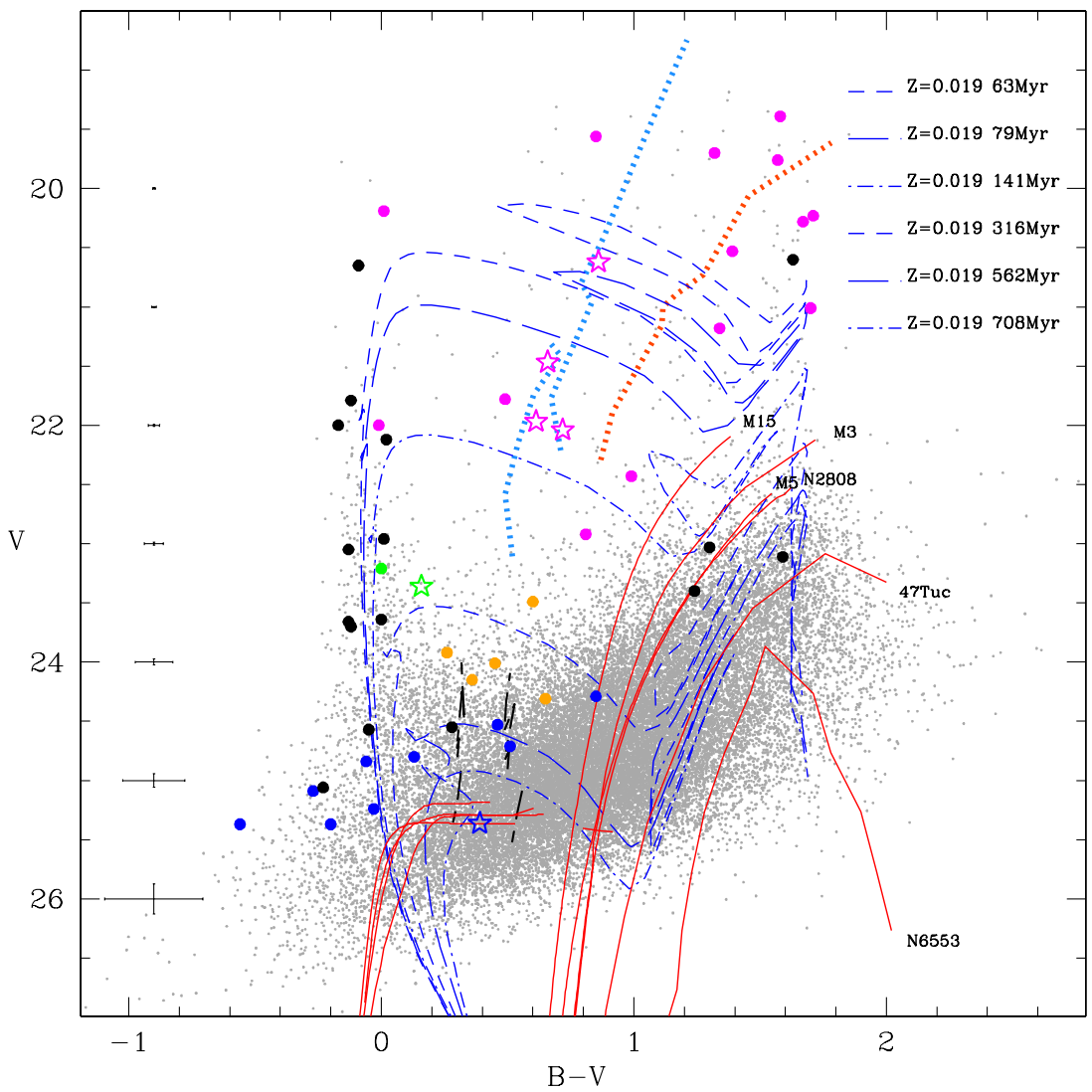

Figure 16. Same as Figure 15, except with isochrones and boundaries of the theoretical IS for the CCs corresponding to $Z=0.019$.

(A color version of this figure is available in the online journal.) 
in the CMD. To account for the various types of variables in each figure we have also overplotted the theoretical ISs of the RR Lyrae stars (Di Criscienzo et al. 2004), ACs with $Z=0.0004$ and $1.3<M<2.2 M_{\odot}$, and CCs, respectively, with $Z=0.008$ and $Z=0.019$. We have then used the mean ridge lines of the GGCs M15, M3, M5, NGC 2808, 47 Tuc, and NGC 6553 (drawn from the $B, V$ database of GGCs by Piotto et al. 2002, from Ferraro et al. 1997 for M3, and from Ortolani et al. 1995 for NGC 6553) to account for the oldest $(t>10 \mathrm{Gyr})$ stellar component. These clusters span a range in metallicity from $[\mathrm{Fe} / \mathrm{H}]=-0.16$ for $\mathrm{NGC} 6553$ to $[\mathrm{Fe} / \mathrm{H}]=-2.33$ for $\mathrm{M} 15$ on the Carretta et al. (2009, hereafter C09) metallicity scale. The observed GGC ridge lines were preferred to theoretical isochrones of corresponding age and metal abundance, since the latter are known to be affected by large uncertainties in the color transformations from the theoretical to the observational plane. To plot ISs, GC ridge lines, and isochrones on the observed CMDs we have assumed $E(B-V)=0.08 \mathrm{mag}, A_{V}=3.315$ $E(B-V)$ and $A_{B}=4.315 E(B-V)$, and $\mu_{0}(\mathrm{M} 31)=24.43 \mathrm{mag}$.

Figures 15 and 16 demonstrate how powerful the approach is of combining information from the isochrone and GC ridge line fitting of the CMD with information obtained from the variable star population present in this region of M31. In fact, the presence of a stellar population as old as $t \geqslant 10 \mathrm{Gyr}$ cannot be unquestionably proven by the comparison of the CMD with the GC ridge lines because of (a) the confusion of stars with different (younger) ages and metal content in the red giant branch region of the CMD as well as some contamination by Galactic red stars, and (b) because the HB of such an old population, if any, cannot be disentangled from the overwhelming young population. However, the mere presence of the RR Lyrae variables having an average magnitude consistent with their membership to M31 definitely proves that such an old population exists and unambiguously traces the HB of such an old population in this region of M31. The properties of the variables also provide hints to the metal abundance of the stars in these regions of M31. In particular, the average magnitude of the RR Lyrae star having full coverage of the light curve (blue star in Figure 15) appears to be consistent with the $\mathrm{HB}$ of the GCs M5 $([\mathrm{Fe} / \mathrm{H}]=-1.33, \mathrm{C} 09)$ and NGC $2808([\mathrm{Fe} / \mathrm{H}]=-1.18$, C09). The red giant branches of these two clusters also fit rather well the upper envelope of the M31 red giant stars, showing that the bulk of the old $(t \geqslant 10 \mathrm{Gyr})$ stellar population in this M31 field has metallicity $[\mathrm{Fe} / \mathrm{H}] \geqslant-1.2 /-1.3$ on the $\mathrm{C} 09$ scale.

As for the younger populations, the CMD isochrone fitting seems to favor a $Z=0.019$ metal abundance. However, the position on the CMD of the variables of a Cepheid type and the comparison with both the isochrones and edges of the theoretical IS for the different metal abundances show that a $Z=0.008$ component is also needed to explain all of the bright variables observed in this field. In fact, although the 9.4 day Cepheid (brightest magenta star) and the candidates with $V \leqslant 21.3$ mag (magenta filled circles in Figures 15 and 16) fall on, or close to, the blue loops of the 63 and $79 \mathrm{Myr}$ isochrones for both the $Z=$ 0.008 and $Z=0.19$, only for $Z=0.008$ do the blue loops extend blueward enough to produce confirmed and candidate CCs with $21.3 \leqslant V \leqslant 22.5 \mathrm{mag}$. Furthermore, the position of the four confirmed CCs with respect to the boundaries of the IS suggests also a lower metal abundance of $Z=0.008$; as for $Z=0.019$, they all lie close to the blue edge of the IS. Particularly at this metallicity, the two bluest Cepheids are located between the blue edge of the fundamental mode and the blue edge of the first overtone mode. This circumstance suggests that if $Z=0.019$, then the two bluest Cepheids are first overtone pulsators, whereas the other two Cepheids are at the fundamental blue edge. Cepheids at the first overtone blue edge are expected to have low pulsation amplitudes. At the same time, the brightest Cepheid is at a luminosity for which only the fundamental mode of pulsation is efficient, so that its proximity to the blue edge again implies a quite low pulsation amplitude (see, e.g., Bono et al. 2000). These predictions are not consistent with the observed pulsation amplitudes that range between 0.84 and $1.29 \mathrm{mag}$ in the $B$ band; they are in better agreement with the values expected if $Z=0.008$ when the Cepheids are in the middle of the IS.

Finally, as anticipated in Section 4.2, the interpretation of the candidates about 1-1.5 mag above the HB (the orange filled circles in Figures 12, 13, and 15) is not easy. Taking into account the magnitude range spanned by these stars ( $V$ in the range from 23.5 to $24.5 \mathrm{mag}$ ) and the periods that are generally shorter than 1 day, with only a couple of exceptions, we have considered three different possibilities: (1) they could be spCCs falling on the short-period tail ( $P<2$ days) of the CCs distribution; (2) they could be ACs tracing an intermediate-age population as metal poor as $[\mathrm{Fe} / \mathrm{H}] \leqslant-1.7$; or $(3)$ they could be overluminous RR Lyrae stars. Although the uncertainty in the periods and the lack of complete light curves for these objects make discerning among the three hypotheses rather difficult, the comparison of the Girardi et al. isochrones shows that the blue loops of the $Z=0.008$ isochrones do not extend blueward enough to cross the IS (see isochrones for 316, 562, and $708 \mathrm{Myr}$ in Figure 15) at the low mass and luminosity of these variables, thus ruling out possibility number 1 , at least for populations with $Z=0.008$. In Figure 17 we show the effect of further reducing the metal abundance to $Z=0.001$ and $Z=0.0004$, respectively. The blue loop of a 708-Myr isochrone with $Z=0.001$ can produce these faint variables at least in part, as well as the brighter CCs, and would produce them even better with $Z=0.0004$. On the other hand, these variables would also be thoroughly consistent with the IS of the ACs for $Z=0.0004$. However, if the bulk of the oldest stellar population in these regions of M31 has a metal abundance of $[\mathrm{Fe} / \mathrm{H}]=-1.2 /-1.3$, as suggested by the RR Lyrae stars and the fitting of the GC HB ridge lines, it does not seem conceivable that the intermediate-age and younger populations in the same field could have metal abundances as low as $Z=0.0004$ (corresponding to $[\mathrm{Fe} / \mathrm{H}]=-1.7$ ). In conclusion, this seems to rule out both the case of the ACs (namely, hypothesis number 2) and, within hypothesis number 1 , the case of the CCs as metal poor as $Z=0.0004$. It is also worth noting that the predicted gap in magnitude between the RR Lyrae level and the faintest Cepheid pulsators at a metallicity of 0.004 is about 0.8 mag (Caputo et al. 2004), which is in excellent agreement with the difference in magnitude between the RR Lyrae and the two AC/spCC pulsators seen in Figures 15, 16 , and 17.

Finally, hypothesis number 3, which states that a large fraction of these variables might be RR Lyrae stars, is supported by their periods being generally below 1 day. However, since these variables also appear to be about 1 mag brighter than the HB of the old population in M31, they are either blended with other sources if they are RR Lyrae stars or they cannot belong to Andromeda but perhaps to a structure (a satellite or the stream) in the galaxy foreground located in the region covered by field S2.

It is obviously too premature to draw any firm conclusions based on only a few variables and the rather incomplete light 


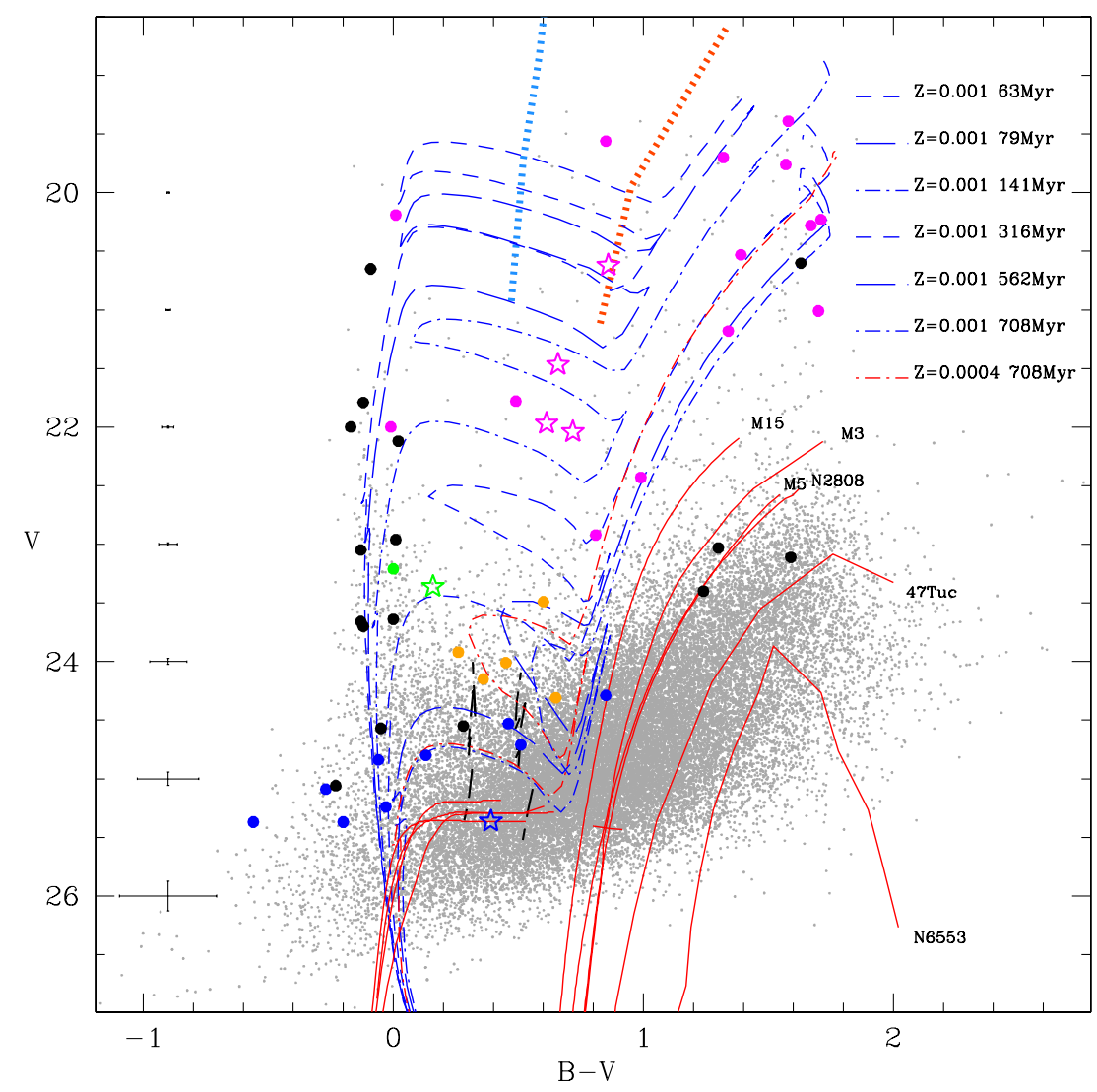

Figure 17. Same as Figures 15 and 16, except with isochrones and boundaries of the theoretical IS for the CCs corresponding to $Z=0.001$. The isochrone with $Z=$ 0.0004 and an age of $708 \mathrm{Myr}$ is shown as a red dot-dashed line.

(A color version of this figure is available in the online journal.)

curves available to us. Still, these results show how much could be learned about the stellar populations and the structure of M31 by combining the study of the galaxy CMD and the variable star properties based on LBT data.

\subsection{Comparison with Previous Variability Studies in M31}

Recent studies of variable stars in M31 include the works of Vilardell et al. (2007) and Joshi et al. (2010) for the Cepheids, and the studies of Brown et al. (2004), Sarajedini et al. (2009), and Jeffery et al. (2011) for the RR Lyrae stars. Both the Brown et al. (2004) and Sarajedini et al. (2009) fields have different size, are rather far away from our LBT pointings, and, generally, are much closer to the M31 disk. These aspects, along with the uncertainty of the completeness of the detection of variable stars in our LBT fields, make the comparison quite difficult. This is particularly true for the RR Lyrae stars since we cannot easily evaluate the completeness of our samples at such faint magnitude levels and, on the other hand, our fields are very peripheral compared with those of Brown et al. (2004) and Sarajedini et al. (2009). Indeed, our two fields sample regions of M31 at the projected distances of $21 \mathrm{kpc}$ (field S2) and $19 \mathrm{kpc}$ (field $\mathrm{H} 1$ ) from the center of the galaxy, respectively, while Brown et al. (2004) observed an HST/ACS field at $11 \mathrm{kpc}$, and Sarajedini et al. (2009) observed two HST/ACS fields at 4 and $6 \mathrm{kpc}$, respectively.

On the other hand, one of the Jeffery et al. (2011) fields, field "halo21," overlaps with our field H1 (see Section 4.2). The comparison of the number density of the RR Lyrae stars in fields H1 and "halo21" shows that, in spite of all of the shortcomings affecting our data, we seem to have reached a good completeness in the detection of these variables.

The comparison is easier for bright variables such as the Cepheids. Vilardell et al. $(2006,2007)$ detected 416 Cepheids in a $33^{\prime} .8 \times 33^{\prime} .8$ field located along the minor axis of M31 (see Figure 1) using the $2.5 \mathrm{~m}$ INT in La Palma, Spain. This corresponds to an average density of 0.36 Cepheids $\operatorname{arcmin}^{-2}$. The Cepheids in the Vilardell et al. sample have a period distribution roughly peaking around 4 days (see Figure 2 in their paper), and they span the magnitude range of $V \sim 23$ to $V \sim 19.2 \mathrm{mag}$, which overlaps well with the range in magnitude spanned by the Cepheids in our LBT fields. Of our LBT pointings, the region imaged in the upper portion of the CCD1 of field S2 is the closest to the M31 disk. In this region we have identified 18 bona fide CCs and another six variables with a more uncertain classification, all having a visual magnitude in the range of 23 to $19 \mathrm{mag}$ (Figure 12). Accounting for the trimming of the $V$ frames, these variables span an area of about $61.44 \mathrm{arcmin}^{2}$, providing a density of 0.39 Cepheids $\operatorname{arcmin}^{-2}$, which is in good agreement with the density findings of Vilardell et al. Unfortunately, we only have a very preliminary estimate of the periods of our Cepheids; thus, it is not possible to make a sound comparison of the period distributions. As for the metal abundances, Vilardell et al. (2007) assume the galactocentric metallicity gradient by Zaritsky et al. (1994) and an empirical metallicity correction of the Cepheid period-luminosity (PL) relation. According to their resulting corrections and to the reported galactocentric distances, we estimate that the Cepheids in their sample have $[\mathrm{O} / \mathrm{H}]$ in the range $-0.2 \div 0.2$. Since we are exploring regions of M31 that are quite external (see Figure 1 
Table 12

Distance Estimates from the CCs

\begin{tabular}{lllcccr}
\hline \hline Name & $P$ & $\mu \mathrm{PL}$ & $\mu \mathrm{Wes}$ & $\mu_{\text {ogle }_{H S T}}$ & $\mu \mathrm{PL02}$ & $\mu$ Wes02 \\
\hline V2 & 9.4 & 24.42 & 24.24 & 24.52 & 24.15 & 24.42 \\
V3 & 3.25 & 24.56 & 24.42 & 24.65 & 24.54 & 24.62 \\
V4 & 5.1 & 24.54 & 24.73 & 24.63 & 24.41 & 24.92 \\
V5 & 2.92 & 24.37 & 24.50 & 24.46 & 24.38 & 24.70 \\
\hline
\end{tabular}

and Column 7 of Table 1), we expect that the metallicity of our Cepheids is close to $[\mathrm{O} / \mathrm{H}]=-0.2$, which implies $Z \sim 0.01$. This is consistent with the metallicities $Z=0.019-0.008$ we infer for CCs brighter than $V \sim 23$ mag as compared with the isochrones from Girardi et al. (2002) discussed in Section 4.4.

As for the comparison with Joshi et al. (2010), those authors used a $1 \mathrm{~m}$ telescope and identified 39 short-period ( $P<15$ days) $\mathrm{CCs}$ in a $13^{\prime} \times 13^{\prime}$ region of the M31 disk located along the semimajor axis on the same side of our field S2 (see Figure 1). This corresponds to a density of 0.23 Cepheids $\operatorname{arcmin}^{-2}$, which is about one-half of the value that we derive in the upper part of CCD1 of field S2. On the other hand, the Joshi et al. sample contains Cepheids that are generally brighter and of a longer period than those seen in our sample. In fact, their period distribution peaks at $\log P \sim 0.9$ and 1.1 days, with periods as short as 3.4 days, and most of their Cepheids are at $\langle R\rangle \sim 20-21 \mathrm{mag}$. Our sample includes Cepheids of a shorter period, but our magnitudes and periods are consistent with their results. Particularly, we find $\langle V\rangle=20.62 \mathrm{mag}$ for the 9.4 day Cepheid (V2, see Table 10) and in the same period range they find consistent $R$ magnitudes, based on a typical $V-R$ color and taking into account the uncertainties related to the reddening corrections.

To derive a distance estimate for the four CCs listed in Table 10 and to confirm that they are M31 members, we have used the theoretical PL and the Wesenheit relations for $Z=$ 0.008 and $Z=0.02$ (see Caputo et al. 2000) and the PL relation adopted by the HST Key Project (Freedman et al. 2001), which has the slope by Udalski et al. (1999) and the zero point based on an assumed distance modulus for the Large Magellanic Cloud (LMC) of 18.5 mag. The resulting individual (from the Wesenheit relations) and mean (from the PL) distances are reported in Table 12 , where $\mu_{\mathrm{PL}}, \mu_{\mathrm{Wes}}, \mu_{\mathrm{OGLE}}{ }_{\mathrm{HST}}, \mu_{\mathrm{PL} 02}$, and $\mu_{\mathrm{Wes} 02}$ are distance moduli derived from the theoretical PL and the Wesenheit relations for $Z=0.008$, the PL relation by Freedman et al. (2001), and the theoretical PL and Wesenheit relations for $Z=0.02$. We consider an uncertainty of $\pm 0.1 \mathrm{mag}$ to the individual distance moduli from the Wesenheit relations, while the average distance modulus obtained from the Udalski et al. (1999) PL is $24.57 \pm 0.2 \mathrm{mag}$. The errors on the estimated distances take into account both the observational errors in $B$ and $V$ and the intrinsic dispersion of the adopted relations. Our value is longer, but it is within the errors consistent with the modulus of McConnachie et al. (2005) transformed to our adopted reddening of $E(B-V)=0.08 \mathrm{mag}, \mu_{0}(\mathrm{M} 31)=$ 24.43 mag.

Vilardell et al. (2007) estimated a distance to M31 of $(m-M)_{0}=24.32 \pm 0.12 \mathrm{mag}$ based on an assumed distance modulus of $18.4 \mathrm{mag}$ for the LMC. The difference in the adopted distance modulus for the LMC combined with the adopted metallicity correction explains most of the discrepancies between their distance estimate and our results.

\section{SUMMARY AND CONCLUSIONS}

We have presented $V, B-V$ CMDs reaching the limiting magnitude $V \sim 26$ mag of two fields of the Andromeda galaxy that were observed with the LBT/LBC-blue camera during the SDT. A number of technical problems during the first phase of the LBT/LBC operation and rather unfavorable weather/seeing conditions hampered our observing campaign, thereby limiting our study of the variable star populations in these M31 fields. Nevertheless, we have identified 274 variable stars using the image subtraction technique and present their differential flux $B$ light curves. For 138 of these variable stars we have also obtained an estimate of the $B, V$ magnitudes that allowed us to plot the variables on the CMDs. By combining the information gathered on the period, magnitude, shape of the light curve, and position on the CMD, we were able to classify 214 variables. They include 127 RR Lyrae stars, 71 short- and intermediate-period Cepheids (periods shorter than 9.4 days), and 16 binary systems. We have compared the CMD and the variable star population of the M31 field closest to the galaxy disk and the giant stream with the sets of isochrones by Girardi et al. (2002) for ages in the range of 63-708 Myr, and metal abundances of $Z=0.0004,0.001,0.008$, and 0.019 to fit the young stellar component, as well as with the mean ridge lines of GGCs spanning a range in metallicity from $[\mathrm{Fe} / \mathrm{H}]=-0.16 \mathrm{dex}$ to $[\mathrm{Fe} / \mathrm{H}]=-2.33 \operatorname{dex}$ (on the $\mathrm{C} 09$ metallicity scale) to account for the oldest $(t>10 \mathrm{Gyr})$ stellar component. The isochrone and GC ridge line fittings and the properties of the variable stars show that the composite stellar population present in this M31 region has a typical metal abundance larger than $[\mathrm{Fe} / \mathrm{H}]=-1.2 /-1.3$ dex for the oldest stellar component and for the young stellar component is in the range of $[\mathrm{Fe} / \mathrm{H}] \sim-1.3$ dex to about solar metallicity. Far from being complete and exhaustive, this study nevertheless demonstrates the powerful approach of combining information from the CMD with information obtained from the variable star population.

LBT is an international collaboration among institutions in the United States, Italy, and Germany. The LBT Corporation partners are: the University of Arizona on behalf of the Arizona university system; the Istituto Nazionale di Astrofisica, Italy; the LBT Beteiligungsgesellschaft, Germany, representing the Max-Planck Society, the Astrophysical Institute Potsdam, and the Heidelberg University; the Ohio State University, and the Research Corporation, on behalf of the University of Notre Dame, the University of Minnesota, and the University of Virginia.

This publication makes use of data products from the Two Micron All Sky Survey, which is a joint project of the University of Massachusetts and the Infrared Processing and Analysis Center/California Institute of Technology, funded by the National Aeronautics and Space Administration and the National Science Foundation. We thank Paolo Montegriffo for the development and maintenance of the GRATIS package. This research was partially supported by PRIN-MIUR-2007JJC53X, PI: F. Matteucci, and by COFIS ASI-INAF I/016/07/0.

\section{REFERENCES}

Alard, C. 2000, A\&AS, 144, 363

Alonso-Garcia, J., Mateo, M., \& Worthey, G. 2004, AJ, 127, 868

Baldacci, L., Rizzi, L., Clementini, G., \& Held, E. V. 2005, A\&A, 431, 1189

Barmby, P., Ashby, M. L. N., Bianchi, L., et al. 2006, ApJ, 650, L45

Beccari, G., Bellazzini, M., Clementini, G., et al. 2007, A\&A, 476, 193 
Bellazzini, M., Cacciari, C., Federici, L., Fusi Pecci, F., \& Rich, M. 2003, A\&A, 405,867

Bertin, A., \& Arnouts, S. 1996, A\&AS, 117, 393

Block, D. L., Bournaud, F., Combes, F., et al. 2006, Nature, 443, 832

Bono, G., Castellani, V., \& Marconi, M. 2000, ApJ, 529, 293

Bono, G., Groenewegen, M. A. T., Marconi, M., \& Caputo, F. 2002, ApJ, 574, L33

Bono, G., Marconi, M., \& Stellingwerf, R. F. 1999, ApJS, 122, 167

Brown, T. M., Beaton, R., Chiba, M., et al. 2008, ApJ, 685, L121

Brown, T. M., Ferguson, H. C., Smith, E., et al. 2003, ApJ, 592, L17

Brown, T. M., Ferguson, H. C., Smith, E., et al. 2004, AJ, 127, 2738

Brown, T. M., Smith, E., Ferguson, H. C., et al. 2006, ApJ, 625, 323

Brown, T. M., Smith, E., Ferguson, H. C., et al. 2007, ApJ, 658, L95

Brown, T. M., Smith, E., Ferguson, H. C., et al. 2009, ApJS, 184, 152

Caputo, F. 1998, A\&ARv, 90, 33

Caputo, F., Castellani, V., Degl'Innocenti, S., Fiorentino, G., \& Marconi, M. 2004, A\&A, 424, 927

Caputo, F., Marconi, M., \& Musella, I. 2000, A\&A, 354, 610

Carretta, E., Bragaglia, A., Gratton, R., D’Orazi, V., \& Lucatello, S. 2009, A\&A, 508,695

Castellani, V., Cignoni, M., Degl'Innocenti, S., Petroni, S., \& Prada Moroni, P. G. 2002, MNRAS, 334, 69

Catelan, M. 2004, in ASP Conf. Ser. 310, Variable Stars in the Local Group, ed. D. W. Kurtz \& K. R. Pollard (San Francisco, CA: ASP), 113

Choi, P. I., Guhathakurta, P., \& Johnston, K. V. 2002, AJ, 124, 310

Cignoni, M., Tosi, M., Bragaglia, A., Kalirai, J. S., \& Davis, D. S. 2008, MNRAS, 386, 2235

Clementini, G. 2010, in Variable Stars, the Galactic halo and Galaxy Formation, ed. C. Sterken, N. Samus, \& L. Szabados (Moscow: Sternberg Astronomical Institute of Moscow Univ.), 107

Clementini, G., Baldacci, L., Bragaglia, A., et al. 2004, in ASP Conf. Ser. 310 , Variable Stars in the Local Group, ed. D. W. Kurtz \& K. R. Pollard (San Francisco, CA: ASP), 60

Clementini, G., Contreras, R., Federici, L., et al. 2009, ApJ, 704, L103

Clementini, G., Di Tomaso, S., Di Fabrizio, L., et al. 2000, AJ, 120, 2054

Clementini, G., Federici, L., Corsi, C., et al. 2001, ApJ, 559, L109

Contreras Ramos, R. 2010, PhD thesis, Univ. of Bologna

Di Criscienzo, M., Greco, C., Ripepi, V., et al. 2011, AJ, 141, 81

Di Criscienzo, M., Marconi, M., \& Caputo, F. 2004, AJ, 612, 1092

Di Fabrizio, L. 1999, Laurea thesis, Univ. Bologna

Dolphin, A. E., Saha, A., Olszewski, E. W., et al. 2004, AJ, 127, 875

Durrell, P. R., Harris, W., \& Pritchet, C. I. 2001, AJ, 121, 2557

Fardal, M. A., Babul, A., Guhathakurta, P., Gilbert, K. M., \& Dodge, C. 2008, ApJ, 382, L33

Ferguson, A. M., Irwin, M. J., Ibata, R. A., Lewis, G. F., \& Tanvir, N. R. 2002, AJ, 124, 1452

Ferraro, F. R., Carretta, E., Corsi, C. E., et al. 1997, A\&A, 320, 757

Fiorentino, G., Limongi, M., Caputo, F., \& Marconi, M. 2006, A\&A, 460, 155

Fiorentino, G., Monachesi, A., Trager, S. C., et al. 2010, ApJ, 708, 817
Font, A. S., Johnston, K. V., Guhathakurta, P., Majewski, S. R., \& Rich, R. M 2006, AJ, 131, 1436

Freedman, W. 1988, ApJ, 326, 691

Freedman, W., Madore, B. F., Gibson, B. K., et al. 2001, ApJ, 553, 47

Giallongo, E., Ragazzoni, R., Grazian, A., et al. 2008, A\&A, 482, 349

Girardi, L., Bertelli, G., Bressan, A., et al. 2002, A\&A, 391, 195

Habing, H. J., Miley, G., Young, E., et al. 1984, ApJ, 278, L59

Ibata, R., Irwin, M., Lewis, G., Ferguson, A. M. N., \& Tanvir, N. 2001, Nature, 412, 49

Ibata, R., Martin, N. F., Irwin, M., et al. 2007, ApJ, 671, 1591

Jeffery, E. J., Smith, E., Brown, T. M., et al. 2011, AJ, 141, 171

Joshi, Y. C., Narasimha, D., Pandey, A. K., \& Sagar, R. 2010, A\&A, 512, A66

Marconi, M., Fiorentino, G., \& Caputo, F. 2004, A\&A, 417, 1101

Martin, N. F., McConnachie, A. W., Irwin, M., et al. 2009, ApJ, 705, 758

Massey, P., Olsen, K. A. G., Hodge, P. W., et al. 2006, AJ, 131, 2478

Mateo, M. 1998, in Stellar Astrophysics for the Local Group, ed. A. Aparicio, A. Herrero, \& F. Sánchez (Cambridge: Cambridge Univ. Press), 407

Mateo, M. 2000, in ASP Conf. Ser. 203, The Impact of Large-Scale Surveys on Pulsating Star Research, ed. L. Szabados \& D. Kurtz (San Francisco, CA: ASP), 187

McConnachie, A. W., Huxor, A., Martin, N. F., et al. 2008, ApJ, 688, 1009

McConnachie, A. W., Irwin, M. J., Ferguson, A. M. N., Ibata, R. A., Lewis, G. F., \& Tanvir, N. 2005, MNRAS, 356, 979

McConnachie, A. W., Irwin, M. J., Ibata, R. A., et al. 2003, MNRAS, 343, 1335

McConnachie, A. W., Irwin, M. J., Ibata, R. A., et al. 2009, Nature, 461, 66

McConnachie, A. W., Irwin, M. J., Lewis, G. F., et al. 2004, MNRAS, 351, L94

Oosterhoff, P. T. 1939, Bull. Astron. Inst. Neth., 9, 11

Ortolani, S., Renzini, A., Gilmozzi, R., et al. 1995, Nature, 377, 701

Piotto, G., King, I. R., Djorgovski, S. G., et al. 2002, A\&A, 391, 945

Pritchet, C. J., \& van den Bergh, S. 1987, ApJ, 316, 517

Richardson, J. C., Ferguson, A. M. N., Johnson, R. A., et al. 2008, AJ, 135, 1998

Richardson, J. C., Irwin, M. J., McConnachie, A. W., et al. 2011, ApJ, 732, 76

Saha, A. 1999, Ap\&SS, 267, 193

Sarajedini, A., Mancone, C. L., Lauer, T. R., et al. 2009, AJ, 138, 184

Schechter, P. L., Mateo, M., \& Saha, A. 1993, PASP, 105, 1342

Schlegel, D. J., Finkbeiner, D. P., \& Davis, M. 1998, ApJ, 500, 525

Stellingwerf, R. F. 1978, ApJ, 224, 953

Stephens, A. W., Frogel, J. A., Freedman, W., et al. 2001, AJ, 121, 2597

Stetson, P. B. 1987, PASP, 99, 191

Stetson, P. B. 1994, PASP, 106, 250

Udalski, A., Szymanski, M., Kubiak, M., et al. 1999, Acta Astron., 49, 201

van den Bergh, S. 2000, The Galaxies of the Local Group (Cambridge: Cambridge Univ. Press)

van den Bergh, S. 2006, in The Local Group as an Astrophysical Laboratory, ed. M. Livio et al. (Cambridge: Cambridge Univ. Press), 1

Vilardell, F., Jordi, C., \& Ribas, I. 2007, A\&A, 473, 847

Vilardell, F., Ribas, I., \& Jordi, C. 2006, A\&A, 459, 321

Wisniewski, W. Z., \& Johnson, H. L. 1968, Commun. Lunar Planet. Lab., 7, 57

Zaritsky, D., Kennicut Jr, R. C., \& Huchra, J. P. 1994, ApJ, 420, 87 